Japan. J. Math.

Vol. 19, No. 2, 1994

\title{
Vector valued Siegel modular forms and their $L$-functions; Application of a differential operator
}

\author{
By Hideshi TAKAYAnagi \\ (Received March 17, 1992) \\ (Revised July 2, 1992)
}

\section{Introduction}

As for automorphic $L$-functions attached to holomorphic Siegel modular forms for $S p(n, \mathbb{Z})(n \geq 2)$ in the sense of Langlands [18, 19], it is known so far that two types of them, namely the spinor type and the standard type, can be continued analytically to the whole complex plane. They are $L$-functions corresponding to the spinor representation of $S O(2 n+1, \mathbb{C})$ and to the standard representation of $S O(2 n+1, \mathbb{C})$ respectively.

Spinor $L$-functions were introduced by Langlands [18] and Andrianov [1] and for scalar valued cases of $n=2$, Andrianov [1] proved their meromorphic continuation and the functional equations. Moreover, their poles were studied by Evdokimov [10] and Oda [23]. For vector valued cases of $n=2$, Arakawa [4] and Sugano [26] have discovered that the spinor $L$-function is continued analytically as an entire function.

On the other hand, standard $L$-functions were also introduced by Langlands [18] and Andrianov [2]. For scalar valued cases, Andrianov-Kalinin [3] (in special cases) and Böcherer [7] (in general; see also Piatetski-Shapiro and Rallis [24]) proved their meromorphic continuation and the functional equations. Recently Mizumoto [21] has obtained results on their poles and residues. We will get the functional equations and poles of standard $L$-functions explicitly for a certain vector valued case (cf. Piatetski-Shapiro and Rallis [24], Weissauer [28]).

Suppose $n$ is a positive integer, $k$ is a positive even integer, $s$ is a complex number such that $k+2 \operatorname{Re}(s)>n+1$, and $Z$ belongs to the Siegel upper half space $\mathfrak{H}_{n}$ (of degree $n$ ). The way of constructing integral representation of the standard $L$-function by the use of Eisenstein series $E_{k}^{n}(Z, s)$ (defined in $\S 1$ ) is based on Böcherer [7] and the method of determining poles by the use of its integral representation is based on Mizumoto [21]. We use the differential operator $L^{k, l}$ (defined in §2) introduced by Böcherer-Satoh-Yamazaki [9] to transform the scalar valued Eisenstein series into a vector valued function. 
In the process of constructing the integral representation, we use the Garrett's pullback formula [12] to decompose $\left(L^{k, l} E_{k}^{2 n}\right)\left(\left(\begin{array}{cc}Z & 0 \\ 0 & W\end{array}\right), s\right), Z, W \in \mathfrak{H}_{n}$, into functions for $Z$ and for $W$. The pullback sends the Eisenstein series $E_{k}^{m+n}\left(\left(\begin{array}{cc}Z & 0 \\ 0 & W\end{array}\right), s\right), Z \in \mathfrak{H}_{m}, W \in \mathfrak{H}_{n}$, to modular forms for $Z$ and for $W$ and it is studied by Garrett [12] for the holomorphic case (i.e., $s=0$ ) and by Böcherer [7] for real analytic cases. Moreover Böcherer-Satoh-Yamazaki [9] deals with the pullback formula of the vector valued function $\left(L^{k, l} E_{k}^{m+n}\right)\left(\left(\begin{array}{cc}Z & 0 \\ 0 & W\end{array}\right), 0\right)$.

The author would like to thank Professor S. Mizumoto who introduced the author to the subject and gave useful advice in the whole process of producing this paper.

\section{Notations.}

$1^{\circ}$. As usual, $\mathbb{Z}$ is the ring of rational integers, $\mathbb{Q}$ the field of rational numbers, $\mathbb{R}$ the field of real numbers, $\mathbb{C}$ the field of complex numbers.

$2^{\circ}$. Let $m, n \in \mathbb{Z}, m, n>0$. If $A$ is an $m \times n$-matrix, then we write it also as $A^{(m, n)}$, and as $A^{(m)}$ if $m=n$. The identity matrix of size $n$ is denoted by $1_{n}$.

$3^{\circ}$. For $m, n \in \mathbb{Z}, m, n>0$, and a commutative ring $R$ containing 1 , let $R^{(m, n)}$ (resp. $R^{(n)}$ ) be the $R$-module of all $m \times n$ (resp. $\left.n \times n\right)$ matrices with entries in $R$.

$4^{\circ}$. For a real symmetric positive definite matrix $S, S^{1 / 2}$ is the unique real symmetric positive definite matrix such that $\left(S^{1 / 2}\right)^{2}=S$.

$5^{\circ}$. For matrices $A^{(m)}, B^{(m, n)}$, we define $A[B]:={ }^{t} \bar{B} A B$, where ${ }^{t} B$ is the transpose of $B$ and $\bar{B}$ is the complex conjugate of $B$.

$6^{\circ}$. For $n \in \mathbb{Z}, n>0$, we put

$$
\begin{aligned}
& \mathbb{T}^{(n)}:=\left\{T=\left(\begin{array}{llll}
t_{1} & & & 0 \\
& t_{2} & & \\
& & \ddots & \\
0 & & & t_{n}
\end{array}\right) \in \mathbb{Z}^{(n)}\right. \\
& \left.\mid t_{i}>0 \text { for each } i \in \mathbb{Z} \text { with } 1 \leq i \leq n, t_{1}|\cdots| t_{n}\right\} \text {. }
\end{aligned}
$$

$7^{\circ}$. For $n \in \mathbb{Z}, n>0$, let $\Gamma^{n}:=S p(n, \mathbb{Z})$ be the Siegel modular group of degree $n$ and let $\mathfrak{H}_{n}$ be the Siegel upper half space of degree $n$, that is,

$$
\mathfrak{H}_{n}:=\left\{Z=X+\left.i Y \in \mathbb{C}^{(n)}\right|^{t} Z=Z, Y>0\right\} .
$$


For $N \in \mathbb{Z}, N>0$, we put

$$
\Gamma_{0}^{n}(N):=\left\{\left(\begin{array}{ll}
A^{(n)} & B^{(n)} \\
C^{(n)} & D^{(n)}
\end{array}\right) \in \Gamma^{n} \mid C \equiv 0 \bmod N\right\}
$$

For each $r \in \mathbb{Z}$ with $0 \leq r \leq n$, we put

$$
P_{n, r}:=\left\{\left(\begin{array}{cc}
* & * \\
C^{(n)} & D^{(n)}
\end{array}\right) \in \Gamma^{n} \mid C=\left(\begin{array}{cc}
0 & 0 \\
0 & C_{4}^{(r)}
\end{array}\right), D=\left(\begin{array}{cc}
* & 0 \\
* & D_{4}^{(r)}
\end{array}\right)\right\}
$$

All these are subgroups of $\Gamma^{n}$.

$8^{\circ}$. For $l \in \mathbb{Z}, l \geq 0$, we put

$$
(a)_{l}:= \begin{cases}a(a+1) \cdots(a+l-1), & \text { if } l>0 \\ 1, & \text { if } l=0\end{cases}
$$

The symbol ()$_{l}$ is called the Pochhammer symbol. Note $(a)_{l}=(-1)^{l}(-a-l+1)_{l}$. We also have

$$
(a+b)_{l}=\sum_{r=0}^{l}\left(\begin{array}{l}
l \\
r
\end{array}\right)(a)_{r}(b)_{l-r}
$$

where $\left(\begin{array}{l}l \\ r\end{array}\right)=\frac{l !}{r !(l-r) !}$ is the binomial coefficient.

$9^{\circ}$. For $n \in \mathbb{Z}, n \geq 0$, we put

$$
\Gamma_{n}(s):=\prod_{j=1}^{n} \Gamma\left(s-\frac{j-1}{2}\right), \quad \Gamma_{\mathbb{R}}(s):=\pi^{-\frac{s}{2}} \Gamma\left(\frac{s}{2}\right), \quad \Gamma_{\mathbb{C}}(s):=2(2 \pi)^{-s} \Gamma(s)
$$

and

$$
\xi(s):=\Gamma_{\mathbb{R}}(s) \zeta(s)=\xi(1-s),
$$

where $\Gamma(s)$ is the gamma function and $\zeta(s)$ is the Riemann zeta function. Throughout the paper we understand that the empty product is equal to 1 .

\section{§1. Preliminary}

\subsection{Vector valued Siegel modular forms}

Let $\rho$ be a finite-dimensional representation of $G L(n, \mathbb{C})$ with a representation space $\mathcal{V}$. By definition, $\mathcal{V}$-valued $C^{\infty}$-Siegel modular forms of weight $\rho$ are $C^{\infty}$ functions from $\mathfrak{H}_{n}$ to $\mathcal{V}$ satisfying

$$
(f \mid M)(Z)=f(Z)
$$


for all $Z \in \mathfrak{H}_{n}$ and $M=\left(\begin{array}{ll}A^{(n)} & B^{(n)} \\ C^{(n)} & D^{(n)}\end{array}\right) \in \Gamma^{n}$, where

$$
(f \mid M)(Z):=\rho\left((C Z+D)^{-1}\right) f(M\langle Z\rangle) \text { and } M\langle Z\rangle:=(A Z+B)(C Z+D)^{-1} \text {. }
$$

The space of all such functions is denoted by $M_{\rho}^{n}(\mathcal{V})^{\infty}$.

A function $f$ from $\mathfrak{H}_{n}$ to $\mathcal{V}$ is called a $\mathcal{V}$-valued Siegel modular form of weight $\rho$ if it satisfies the following properties:

$$
\text { holomorphic on } \mathfrak{H}_{n}
$$

$$
\text { for all } M \in \Gamma^{n},(f \mid M)(Z)=f(Z)
$$

$$
\text { holomorphic at the cusps if } n=1 \text {. }
$$

The space of $\mathcal{V}$-valued Siegel modular forms of weight $\rho$ is denoted by $M_{\rho}^{n}(\mathcal{V})$.

We define the Siegel operator $\Phi$ on $M_{\rho}^{n}(\mathcal{V})$ by

$$
(\Phi f)(Z):=\lim _{t \rightarrow \infty} f\left(\left(\begin{array}{cc}
Z & 0 \\
0 & i t
\end{array}\right)\right)
$$

for $Z \in \mathfrak{H}_{n-1}$. Let $\mathcal{V}^{\prime}$ be the subspace of $\mathcal{V}$ generated by the values of $\Phi f$ for all $f \in M_{\rho}^{n}(\mathcal{V})$. Then $\mathcal{V}^{\prime}$ is invariant under the transformation

$$
\rho\left(\left(\begin{array}{ll}
g & 0 \\
0 & 1
\end{array}\right)\right), g \in G L(n-1, \mathbb{C})
$$

If we assume $\mathcal{V}^{\prime} \neq\{0\}$, we get the representation $\rho^{\prime}$ of $G L(n-1, \mathbb{C})$ with the representation space $\mathcal{V}^{\prime}$. Thus the operator $\Phi$ defines the map

$$
\Phi: M_{\rho}^{n}(\mathcal{V}) \longrightarrow M_{\rho^{\prime}}^{n-1}\left(\mathcal{V}^{\prime}\right)
$$

Suppose $f \in M_{\rho}^{n}(\mathcal{V})$. Then it is called a cusp form if it satisfies $\Phi f=0$, and we put

$$
S_{\rho}^{n}(\mathcal{V}):=\left\{f \in M_{\rho}^{n}(\mathcal{V}) \mid f \text { is a cuspform }\right\} .
$$

Each $f \in M_{\rho}^{n}(\mathcal{V})$ has a Fourier expansion

$$
f(Z)=\sum_{H \geq 0} a(H) \exp (2 \pi i \operatorname{trace}(H Z)), a(H) \in \mathcal{V},
$$

with $H$ running over all symmetric positive semi-definite semi-integral matrices of size $n$. If $f \in S_{\rho}^{n}(\mathcal{V}), H$ runs over all symmetric positive definite semi-integral matrices. 
Let $\mathcal{V} \cong \oplus \mathcal{V}_{i}$ be a decomposition of $\mathcal{V}$ into a direct sum of irreducible representations. Then we get a decomposition

$$
M_{\rho}^{n}(\mathcal{V}) \cong \oplus M_{\rho_{i}}^{n}\left(\mathcal{V}_{i}\right)
$$

where for each $i, \rho_{i}$ is a representation of $G L(n, \mathbb{C})$ with the representation space $\mathcal{V}_{i}$.

If $\rho$ is an irreducible rational representation, $\rho$ is equivalent to an irreducible rational representation $\tilde{\rho}$ satisfying the following condition: Let $\tilde{\mathcal{V}}$ be the representation space of $\tilde{\rho}$. Then, there exists a unique one-dimensional vector subspace $\mathbb{C} \tilde{v}$ of $\tilde{\mathcal{V}}$ such that for any upper triangular matrix of $G L(n, \mathbb{C})$,

$$
\tilde{\rho}\left(\left(\begin{array}{ccc}
g_{11} & & * \\
& \ddots & \\
0 & & g_{n n}
\end{array}\right)\right) \tilde{v}=\left(\prod_{j=1}^{n} g_{j j}^{\lambda_{j}}\right) \tilde{v}
$$

where $\left(\lambda_{1}, \lambda_{2}, \cdots, \lambda_{n}\right) \in \mathbb{Z}^{n}$ and $\lambda_{1} \geq \lambda_{2} \geq \cdots \geq \lambda_{n}$.

Then we write

$$
\rho \sim\left(\lambda_{1}, \lambda_{2}, \cdots, \lambda_{n}\right)
$$

Suppose $\rho \sim\left(\lambda_{1}, \lambda_{2}, \cdots, \lambda_{n}\right)$. We note that $M_{\rho}^{n}(\mathcal{V})=\{0\}$ if $\lambda_{n}<0$ and that $M_{\rho}^{n}(\mathcal{V})^{\infty}=\{0\}$ if $\lambda_{1}+\cdots+\lambda_{n} \not \equiv 0 \bmod 2$.

Let $k, l \in \mathbb{Z}, k>0, l \geq 0$. For a vector space $W$, we denote by $\operatorname{sym}^{l}(W)$ its $l$-th symmetric tensor product. We identify $\operatorname{sym}^{0}(W)$ with $\mathbb{C}$. Let $x=\left(x_{1}, \cdots, x_{n}\right)$ be a row vector consisting of $n$ indeterminates. Then we put $V=\mathbb{C} x_{1} \oplus \cdots \oplus \mathbb{C} x_{n}$. We identify $\operatorname{sym}^{l}(V)$ with the subset of $\mathbb{C}\left[x_{1}, \cdots, x_{n}\right]$ which consists of the homogeneous polynomials of degree $l$. Let $\rho$ be a representation $\operatorname{det}^{k} \otimes \operatorname{sym}^{l}$ of $G L(n, \mathbb{C})$ with a representation space $\operatorname{sym}^{l}(V)$. Then for each $g \in G L(n, \mathbb{C}), \rho(g)$ acts on $\operatorname{sym}^{l}(V)$ by

$$
\rho(g)(v(x))=\operatorname{det} g^{k} v(x g), \quad v=v(x) \in \operatorname{sym}^{l}(V) .
$$

We note that $\rho \sim(k+l, k, \cdots, k)$ and $\operatorname{dim}\left(\operatorname{sym}^{l}(V)\right)=\left(\begin{array}{c}n+l-1 \\ l\end{array}\right)$.

From now on, we put $\rho=\operatorname{det}^{k} \otimes \operatorname{sym}^{l}$ unless stated otherwise.

We write $M_{k, l}^{n}\left(\operatorname{sym}^{l}(V)\right)^{\infty}, \quad M_{k, l}^{n}\left(\operatorname{sym}^{l}(V)\right)$ and $S_{k, l}^{n}\left(\operatorname{sym}^{l}(V)\right)$ for $M_{\rho}^{n}\left(\operatorname{sym}^{l}(V)\right)^{\infty}, M_{\rho}^{n}\left(\operatorname{sym}^{l}(V)\right)$ and $S_{\rho}^{n}\left(\operatorname{sym}^{l}(V)\right)$, respectively.

REMARKs. (i) For $n=2$, any finite-dimensional rational representation of $G L(n, \mathbb{C})$ is equivalent to a direct sum of representations $\rho=\operatorname{det}^{k} \otimes \operatorname{sym}^{l}$ for $k, l \in \mathbb{Z}$.

(ii) If $n k \not \equiv l \bmod 2, M_{k, l}^{n}\left(\operatorname{sym}^{l}(V)\right)^{\infty}=\{0\}$.

As above, let $V=\mathbb{C} x_{1} \oplus \cdots \oplus \mathbb{C} x_{n}, x=\left(x_{1}, \cdots, x_{n}\right)$. 
For $\sum_{j=1}^{n} a_{j} x_{j}, \sum_{j=1}^{n} b_{j} x_{j} \in V$, we define an inner product of them by

$$
\left\langle\sum_{j=1}^{n} a_{j} x_{j}, \sum_{j=1}^{n} b_{j} x_{j}\right\rangle:=\sum_{j=1}^{n} a_{j} \bar{b}_{j} .
$$

The inner product induces an inner product of $\operatorname{sym}^{l}(V)$ defined by

$$
\left\langle v_{1} \cdots v_{l}, w_{1} \cdots w_{l}\right\rangle:=\frac{1}{l !} \sum_{\tau \in \mathfrak{S}_{l}} \prod_{j=1}^{l}\left\langle v_{\tau(j)}, w_{j}\right\rangle
$$

where $v_{j}, w_{j} \in V$. It is also denoted by $\langle, \quad\rangle$. Then, for $v=v(x), w=w(x) \in$ $\operatorname{sym}^{l}(V)$, the inner product has the following properties:

$$
\langle v(x), w(x)\rangle=\overline{\langle w(x), v(x)\rangle}
$$

$$
\begin{aligned}
\left\langle\rho(g) v(x), \rho\left(g^{\prime}\right) w(x)\right\rangle & =\left\langle\rho\left({ }^{t} \bar{g}^{\prime} g\right) v(x), w(x)\right\rangle \\
& =\left\langle v(x), \rho\left({ }^{t} \bar{g} g^{\prime}\right) w(x)\right\rangle
\end{aligned}
$$

for $g, g^{\prime} \in G L(n, \mathbb{C})$, and

$$
\langle\rho(U) v(x), \rho(U) w(x)\rangle=\langle v(x), w(x)\rangle
$$

for any $U \in U(n, \mathbb{C})$.

Suppose $f, g \in M_{k, l}^{n}\left(\operatorname{sym}^{l}(V)\right)^{\infty}$. The Petersson inner product of $f$ and $g$ is defined by

$$
\begin{gathered}
(f, g):=\int_{\Gamma^{n} \backslash \mathfrak{H}_{n}}\langle\rho(\sqrt{\operatorname{Im}(W)}) f(W), \rho(\sqrt{\operatorname{Im}(W)}) g(W)\rangle \\
\times \operatorname{det}(\operatorname{Im}(W))^{-n-1} d X d Y
\end{gathered}
$$

if the right-hand side is convergent. Here $W=X+i Y$ with real matrices $X=\left(x_{j h}\right)$ and $Y=\left(y_{j h}\right)$;

$$
d X:=\prod_{j \leq h} d x_{j h}, \quad d Y:=\prod_{j \leq h} d y_{j h}
$$

the integral is taken over a fundamental domain of $\Gamma^{n} \backslash \mathfrak{H}_{n}$. We write $d W=d X d Y$ when there is no fear of confusion. 


\subsection{Hecke algebra and Hecke operator}

We put

$$
\begin{aligned}
& \Delta^{n}:=G^{+} S p(n, \mathbb{Q})
\end{aligned}
$$

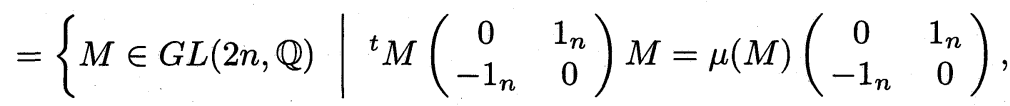

$$
\begin{aligned}
& \mu(M)>0\}
\end{aligned}
$$

and for a prime number $p$

$$
\Delta_{p}^{n}:=\Delta^{n} \cap G L\left(2 n, \mathbb{Z}\left[\frac{1}{p}\right]\right) .
$$

Let $\mathcal{H}\left(\right.$ resp. $\mathcal{H}_{p}$ ) be a free $\mathbb{C}$-module generated by the double cosets $\Gamma^{n} g \Gamma^{n}$, $g \in \Delta^{n}$ (resp. $\Delta_{p}^{n}$ ). Then $\mathcal{H}$ is a commutative algebra and $\mathcal{H}=\otimes_{p} \mathcal{H}_{p}$. Moreover, the structure of $\mathcal{H}_{p}$ is known: For $1 \leq j \leq n$, let $w_{j}$ be an automorphism of $\mathbb{C}\left[X_{0}^{ \pm 1}, \cdots, X_{n}^{ \pm 1}\right]$ such that

$$
w_{j}\left(X_{0}\right)=X_{0} X_{j}, w_{j}\left(X_{j}\right)=X_{j}^{-1} \text { and } w_{j}\left(X_{i}\right)=X_{i}(1 \leq i \leq n, i \neq j) .
$$

The automorphisms $w_{j}(1 \leq j \leq n)$ and the symmetric group $\mathfrak{S}_{n}$ consisting of permutations of $X_{i}(1 \leq i \leq n)$ generate a finite group $W$. We denote by $\mathbb{C}\left[X_{0}{ }^{ \pm 1}, \cdots, X_{n}{ }^{ \pm 1}\right]^{W}$ the $W$-invariant subalgebra of $\mathbb{C}\left[X_{0}{ }^{ \pm 1}, \cdots, X_{n}{ }^{ \pm 1}\right]$. Then

$$
\mathcal{H}_{p} \cong \mathbb{C}\left[X_{0}^{ \pm 1}, \cdots, X_{n}^{ \pm 1}\right]^{W}
$$

where the isomorphism can be written explicitly.

For $g \in \Delta_{n}$, let $\Gamma^{n} g \Gamma^{n}=\bigcup_{i=1}^{r} \Gamma^{n} g_{i}$ be the decomposition of $\Gamma^{n} g \Gamma^{n}$ into left cosets.

For $f \in M_{k, l}^{n}\left(\operatorname{sym}^{l}(V)\right)\left(\operatorname{resp} . S_{k, l}^{n}\left(\operatorname{sym}^{l}(V)\right), M_{k, l}^{n}\left(\operatorname{sym}^{l}(V)\right)^{\infty}\right)$, we put

$$
f \mapsto f\left|\left(\Gamma^{n} g \Gamma^{n}\right):=\sum_{i=1}^{r} f\right| g_{i}
$$

Then we get a homomorphism

$$
\mathcal{H} \longrightarrow \operatorname{End}\left(M_{k, l}^{n}\left(\operatorname{sym}^{l}(V)\right)\right)
$$

$\left(\right.$ resp. $\left.\operatorname{End}\left(S_{k, l}^{n}\left(\operatorname{sym}^{l}(V)\right)\right), \operatorname{End}\left(M_{k, l}^{n}\left(\operatorname{sym}^{l}(V)\right)^{\infty}\right)\right)$.

Suppose $f \in M_{k, l}^{n}\left(\operatorname{sym}^{l}(V)\right)$ is an eigenform, i.e., a non-zero common eigenfunction of the Hecke algebra. It follows from 


$$
\Gamma^{n} g \Gamma^{n} \mapsto f \mid\left(\Gamma^{n} g \Gamma^{n}\right)=\lambda\left(\Gamma^{n} g \Gamma^{n}\right) f
$$

that a homomorphism

$$
\lambda: \mathcal{H}_{p} \stackrel{\cong}{\longrightarrow}\left[X_{0}^{ \pm 1}, \cdots, X_{n}^{ \pm 1}\right]^{W} \stackrel{\left(X_{0}, \cdots, X_{n}\right) \mapsto\left(\alpha_{0, p}, \cdots, \alpha_{n, p}\right)}{\longrightarrow} \mathbb{C}
$$

with $\left(\alpha_{0, p}, \cdots, \alpha_{n, p}\right) \in\left(\mathbb{C}^{* n+1}\right)^{W}$, is defined. The numbers $\alpha_{0, p}, \cdots, \alpha_{n, p}$ are uniquely determined modulo $W$ and they are called the Satake $p$-parameters of $f$.

\subsection{Standard $L$-function}

Let $f \in S_{k, l}^{n}\left(\operatorname{sym}^{l}(V)\right)$ be an eigenform. We define the standard $L$-function by

$$
D_{f}(s):=\prod_{p}\left\{\left(1-p^{-s}\right) \prod_{i=1}^{n}\left(1-\alpha_{i, p}^{-1} p^{-s}\right)\left(1-\alpha_{i, p} p^{-s}\right)\right\}^{-1},
$$

where $p$ runs over all prime numbers. The right-hand side of (1.3) converges absolutely and locally uniformly for $\operatorname{Re}(s)>n+1$.

We also define the following series:

$$
L(s, f):=\sum_{T \in \mathbb{T}^{(n)}} \lambda(f, T) \operatorname{det}(T)^{-s}
$$

where $\lambda(f, T)$ is an eigenvalue on $f$ of the Hecke operator $\Gamma^{n}\left(\begin{array}{cc}T & 0 \\ 0 & T^{-1}\end{array}\right) \Gamma^{n}, T \in$ $\mathbb{T}^{(n)}$. The right-hand side of (1.4) also converges absolutely and locally uniformly for $\operatorname{Re}(s)>2 n+1$. By Böcherer [8], we have:

$$
\zeta(s) \prod_{i=1}^{n} \zeta(2 s-2 i) L(s, f)=D_{f}(s-n) .
$$

Here we mention a fact on poles of standard $L$-functions, (1.3.1) below:

Let $H_{m, n}(\rho)$ be the (finite-dimensional) $\mathbb{C}$-vector space of polynomial maps

$$
P: \mathbb{C}^{(m, n)} \longrightarrow \operatorname{sym}^{l}(V)
$$

satisfying the following two conditions:

$$
\begin{gathered}
\operatorname{det} g^{m / 2} P(X g)=\rho\left({ }^{t} g\right) P(X) \text { for all } g \in G L(n, \mathbb{C}), X \in \mathbb{C}^{(m, n)}, \\
\sum_{j=1}^{m} \frac{\partial}{\partial x_{j i}} \frac{\partial}{\partial x_{j k}} P(X)=0 \quad \text { for } X=\left(x_{i j}\right) .
\end{gathered}
$$


Such polynomials are called pluriharmonic.

Let $\mathcal{S}_{m}$ be the set of all symmetric positive definite even integral unimodular matrices of size $m$. As is well-known, $\mathcal{S}_{m} \neq \emptyset$ if and only if $m \equiv 0 \bmod 8$. For each $P \in H_{m, n}(\rho)$ and $S \in \mathcal{S}_{m}$ with $m \equiv 0 \bmod 8$, we attach the theta series

$$
\vartheta_{S, P}(Z):=\sum_{G \in \mathbb{Z}^{(m, n)}} P\left(S^{1 / 2} G\right) \exp (\pi i \operatorname{trace}(S[G] Z))
$$

which converges for all $Z \in \mathfrak{H}_{n}$ and belongs to $M_{k, l}^{n}\left(\operatorname{sym}^{l}(V)\right)$. We denote by $B_{k, l}^{n}(m)$ the subspace of $M_{k, l}^{n}\left(\operatorname{sym}^{l}(V)\right)$ of all finite sums of theta series $\vartheta_{S, P}$. The space $B_{k, l}^{n}(m)$ is invariant under the action of the Hecke algebra. We note that $B_{k, l}^{n}(m)=$ $\{0\}$ if $m \not \equiv 0 \bmod 8$ or $m>2 k$.

Under the notation above, we have the following by Weissauer [28]:

(1.3.1) For $n, k, l \in \mathbb{Z}, n, k, l>0$, let $f \in S_{k, l}^{n}\left(\operatorname{sym}^{l}(V)\right)$ be an eigenform. Suppose $k>n$. Then $D_{f}(s)$ has a simple pole at $s=1$ if and only if $f \in B_{k, l}^{n}(2 n) \cap$ $S_{k, l}^{n}\left(\operatorname{sym}^{l}(V)\right)$.

\subsection{Eisenstein series}

For scalar valued cases (i.e., $l=0$ ), we write $M_{k}^{n \infty}, M_{k}^{n}$ and $S_{k}^{n}$ for $M_{k, 0}^{n}\left(\operatorname{sym}^{0}(V)\right)^{\infty}, M_{k, 0}^{n}\left(\operatorname{sym}^{0}(V)\right)$ and $S_{k, 0}^{n}\left(\operatorname{sym}^{0}(V)\right)$, respectively.

For $k \in 2 \mathbb{Z}, k>0, s \in \mathbb{C}$ and $Z \in \mathfrak{H}_{n}$, we define the Eisenstein series by

$$
\begin{gathered}
E_{k}^{n}(Z, s):=\sum_{M=\left(\begin{array}{cc}
* & * \\
C^{(n)} & D^{(n)}
\end{array}\right) \in P_{n, 0} \backslash \Gamma^{n}} \operatorname{det}(C Z+D)^{-k} \operatorname{det}(\operatorname{Im}(M\langle Z\rangle))^{s} .
\end{gathered}
$$

Then $E_{k}^{n}(Z, s) \in M_{k}^{n \infty}$. The function $E_{k}^{n}(Z, s) \operatorname{det}(\operatorname{Im}(Z))^{-s}$ converges absolutely and locally uniformly for $k+2 \operatorname{Re}(s)>n+1$. Moreover we have the following by Mizumoto [21, 22] (see also Andrianov-Kalinin [3], Kalinin [15], Langlands [20]):

(1.4.1) Let $n, k \in \mathbb{Z}, n, k>0, k:$ even. Then for $Z \in \mathfrak{H}_{n}$,

$$
\mathbb{E}_{k}^{n}(Z, s):=\frac{\Gamma_{n}\left(s+\frac{k}{2}\right)}{\Gamma_{n}(s)} \xi(2 s) \prod_{i=1}^{\left[\frac{n}{2}\right]} \xi(4 s-2 i) E_{k}^{n}\left(Z, s-\frac{k}{2}\right)
$$

is invariant under $s \mapsto \frac{n+1}{2}-s$ and it is an entire function in $s$.

It is also known that every partial derivative (in $z_{j h}$ 's) of the Eisenstein series $E_{k}^{n}(Z, s)$ is slowly increasing (locally uniformly in $s$ ). That is, by Mizumoto [22] we have:

(1.4.2) Let $n, k \in \mathbb{Z}, n, k>0, k:$ even. 
For each $s_{0} \in \mathbb{C}$, we can take $d \in \mathbb{Z}, d>0$ and a suitable neighborhood $\mathcal{U}$ of $s_{0}$ depending only on $n, k$ and $s_{0}$ such that $\left(s-s_{0}\right)^{d} E_{k}^{n}(Z, s)$ is holomorphic in $s$ on $\mathcal{U}$. Then, for $s \in \mathcal{U}, l \in \mathbb{Z}, l \geq 0, \operatorname{Im}(Z) \geq \varepsilon 1_{n}(\varepsilon>0)$, there exist positive constants $\alpha, \beta$ depending only on $n, k, l, \varepsilon, s_{0}, d$ and $\mathcal{U}$ such that

$$
\left|\left(s-s_{0}\right)^{d} \frac{\partial^{l}}{\partial z_{j_{1} h_{1}} \cdots \partial z_{j_{l} h_{l}}} E_{k}^{n}(Z, s)\right| \leq \alpha \operatorname{det}(\operatorname{Im}(Z))^{\beta}
$$

$\left(1 \leq j_{\nu}, h_{\nu} \leq n\right)$

Here we summarize some facts on standard $L$-functions attached to scalar valued Siegel modular forms.

Let $f \in S_{k}^{n}$ be an eigenform. Then we put

$$
\Lambda(s, f):=\Gamma_{\mathbb{R}}(s+\varepsilon) \prod_{j=1}^{n} \Gamma_{\mathbb{C}}(s+k-j) D_{f}(s)
$$

with

$$
\varepsilon:= \begin{cases}0 & \text { for } n \text { even } \\ 1 & \text { for } n \text { odd }\end{cases}
$$

Moreover we put

$$
\gamma(s):= \begin{cases}\frac{\Gamma_{n}\left(\frac{s+n}{2}\right)}{\Gamma_{n}\left(\frac{s}{2}\right)} & \text { for } n \text { even } \\ \frac{\Gamma_{n-1}\left(\frac{s+n}{2}\right)}{\Gamma_{n-1}\left(\frac{s-1}{2}\right)} & \text { for } n \text { odd }\end{cases}
$$

We note

$$
\gamma(s)=\gamma(1-s)
$$

Then we have the following ([3], [7], [24]):

(1.4.3) Let $f \in S_{k}^{n}$ be an eigenform with a positive integer $n$ and a positive even integer $k$.

(i) We obtain the integral representation

$$
\left(f, \mathbb{E}_{k}^{2 n}\left(\left(\begin{array}{cc}
-\bar{Z} & 0 \\
0 & *
\end{array}\right), \frac{\bar{s}+n}{2}\right)\right)=2 i^{n k} \pi^{-\frac{1}{2} n^{2}+k n+\frac{1}{2} \varepsilon} \gamma(s) \Lambda(s, f) f(Z) .
$$


(ii) $\Lambda(s, f)$ has a meromorphic continuation to the whole s-plane and satisfies the functional equation

$$
\Lambda(s, f)=\Lambda(1-s, f)
$$

To talk about poles of $\Lambda(s, f)$ we write $H_{m, n}(k)$ and $B_{k}^{n}(m)$ for $H_{m, n}\left(\operatorname{det}^{k} \otimes\right.$ $\operatorname{sym}^{0}$ ) and $B_{k, 0}^{n}(m)$, where we must replace the condition (ii) of $H_{m, n}(\rho)$ in $\S 1.3$ with the condition

$$
\sum_{i=1}^{m} \sum_{j=1}^{n} \frac{\partial^{2}}{\partial x_{i j}^{2}} P(X)=0 \text { for } X=\left(x_{i j}\right)
$$

By Mizumoto [21], we have:

(1.4.4) For $n, k \in \mathbb{Z}, n, k>0$, let $f \in S_{k}^{n}$ be an eigenform. Suppose $k \geq n$. Then $\Lambda(s, f)$ is holomorphic except for possible simple poles at $s=0$ and $s=1$; it has a pole at $s=0$ if and only if $f \in B_{k}^{n}(2 n) \cap S_{k}^{n}$.

\section{§2. Differential operator and Pullback formula}

\subsection{Differential operator}

In what follows, we put

$$
\begin{aligned}
V & =\mathbb{C} x_{1} \oplus \cdots \oplus \mathbb{C} x_{2 n}, & x & =\left(x_{1}, \cdots, x_{2 n}\right), \\
V_{1} & =\mathbb{C} x_{1} \oplus \cdots \oplus \mathbb{C} x_{n}, & e_{1} & =\left(x_{1}, \cdots, x_{n}\right), \\
V_{2} & =\mathbb{C} x_{n+1} \oplus \cdots \oplus \mathbb{C} x_{2 n}, & e_{2} & =\left(x_{n+1}, \cdots, x_{2 n}\right) .
\end{aligned}
$$

Let $\iota$ be an isomorphism from $V_{1}$ to $V_{2}$ defined by $\iota\left(x_{i}\right)=x_{n+i}(1 \leq i \leq n)$. It induces an isomorphism (also denoted by $\iota$ ) from $\operatorname{sym}^{l}\left(V_{1}\right)$ to $\operatorname{sym}^{l}\left(V_{2}\right)$. For $f \in C^{\infty}\left(\mathfrak{H}_{n}, \operatorname{sym}^{l}\left(V_{1}\right)\right)$ we define $\iota(f)$ by

$$
(\iota(f))(Z):=\iota(f(Z))
$$

Let $Z=\left(z_{j h}\right)$ be a variable on $\mathfrak{H}_{2 n}$. For an integer $l \geq 0$ and a function $f \in C^{\infty}\left(\mathfrak{H}_{2 n}, \operatorname{sym}^{l}(V)\right)$, we put

$$
D f:=\left(\frac{\partial}{\partial Z} f\right)\left[{ }^{t} x\right] \quad \text { with } \frac{\partial}{\partial Z}=\left(\frac{1+\delta_{j h}}{2} \frac{\partial}{\partial z_{j h}}\right)_{1 \leq j, h \leq 2 n} .
$$

Here, for $z_{j h}=x_{j h}+i y_{j h}$ we put

$$
\frac{\partial}{\partial z_{j h}}=\frac{1}{2}\left(\frac{\partial}{\partial x_{j h}}-i \frac{\partial}{\partial y_{j h}}\right), \quad \frac{\partial}{\partial \bar{z}_{j h}}=\frac{1}{2}\left(\frac{\partial}{\partial x_{j h}}+i \frac{\partial}{\partial y_{j h}}\right) .
$$


Then $D f$ is a $V^{(l+2)}$-valued function. We also put $D_{\uparrow} f:=\left.D f\right|_{e_{2}=0}, D_{\downarrow} f:=\left.D f\right|_{e_{1}=0}$ and $D_{0}:=D-D_{\uparrow}-D_{\downarrow}$. For a function $f$ on $\mathfrak{H}_{2 n},\left(\begin{array}{ll}Z^{(n)} & { }^{t} U^{(n)} \\ U^{(n)} & W^{(n)}\end{array}\right) \in \mathfrak{H}_{2 n}$, we define the pullback $d^{*}$ by

$$
\left(d^{*} f\right)\left(\left(\begin{array}{cc}
Z & { }^{t} U \\
U & W
\end{array}\right)\right):=f\left(\left(\begin{array}{cc}
Z & 0 \\
0 & W
\end{array}\right)\right)
$$

For $k, l \in \mathbb{Z}, k, l \geq 0$, we define an operator

$$
L^{k, l}: C^{\infty}\left(\mathfrak{H}_{2 n}, \mathbb{C}\right) \longrightarrow C^{\infty}\left(\mathfrak{H}_{n} \times \mathfrak{H}_{n}, \operatorname{sym}^{2 l}(V)\right)
$$

by

$$
L^{k, l}:=\frac{1}{(2 \pi i)^{l}(k)_{l}} d^{*} \sum_{\nu=0}^{\left[\frac{l}{2}\right]} \frac{1}{\nu !(l-2 \nu) !(2-k-l)_{\nu}}\left(D_{\uparrow} D_{\downarrow}\right)^{\nu} D_{0}{ }^{l-2 \nu}
$$

We also define an operator

$$
L_{*}^{k, l}: C^{\infty}\left(\mathfrak{H}_{2 n}, \mathbb{C}\right) \longrightarrow C^{\infty}\left(\mathfrak{H}_{2 n}, \operatorname{sym}^{2 l}(V)\right)
$$

by

$$
L_{*}^{k, l}:=\frac{1}{(2 \pi i)^{l}(k)_{l}} \sum_{\nu=0}^{\left[\frac{l}{2}\right]} \frac{1}{\nu !(l-2 \nu) !(2-k-l)_{\nu}}\left(D_{\uparrow} D_{\downarrow}\right)^{\nu} D_{0}^{l-2 \nu} .
$$

Then we get the following by [9]:

(2.1.1) (i) For $k, l \in \mathbb{Z}, k, l \geq 0, L^{k, l}$ satisfies

$$
\begin{aligned}
d^{*} & \sum_{l=0}^{\infty} \frac{t^{l}}{(2 \pi i)^{l} l !(k)_{l}} D^{l} \\
\quad= & \sum_{l=0}^{\infty}\left(\sum_{\lambda=0}^{\infty} \frac{t^{\lambda}}{(2 \pi i)^{\lambda} \lambda !(k+l)_{\lambda}} D_{\uparrow}^{\lambda}\right)\left(\sum_{\lambda=0}^{\infty} \frac{t^{\lambda}}{(2 \pi i)^{\lambda} \lambda !(k+l)_{\lambda}} D_{\downarrow}^{\lambda}\right) t^{l} L^{k, l} .
\end{aligned}
$$

(ii) The operator $L^{k, l}$ is an operator which sends modular forms to modular forms:

$$
L^{k, l}: M_{k}^{2 n}{ }^{\infty} \longrightarrow M_{k, l}^{n}\left(\operatorname{sym}^{l}\left(V_{1}\right)\right)^{\infty} \otimes M_{k, l}^{n}\left(\operatorname{sym}^{l}\left(V_{2}\right)\right)^{\infty} .
$$

Moreover, $L^{k, l}$ is a holomorphic operator and it satisfies

$$
L^{k, l}: M_{k}^{2 n} \longrightarrow M_{k, l}^{n}\left(\operatorname{sym}^{l}\left(V_{1}\right)\right) \otimes M_{k, l}^{n}\left(\operatorname{sym}^{l}\left(V_{2}\right)\right)
$$


REMARK. On $C^{\infty}$-functions the operators $d^{*}, D_{\uparrow}, D_{\downarrow}$ are commutative and $D, D_{0}, D_{\uparrow}, D_{\downarrow}$ are also commutative. But $d^{*}$ and $D, d^{*}$ and $D_{0}$ are not commutative, respectively. Therefore, $L^{k, l}$ is commutative with $D_{\uparrow}$ and $D_{\downarrow}$, and not commutative with $D$ or $D_{0}$.

The main results of this section is the following:

Proposition 1. Let $k, l \in 2 \mathbb{Z}, k, l>0, s \in \mathbb{C}$ and $k+2 \operatorname{Re}(s)>2 n+1$. For $\mathfrak{Z}=\left(\begin{array}{ll}Z^{(n)} & { }^{t} U^{(n)} \\ U^{(n)} & W^{(n)}\end{array}\right) \in \mathfrak{H}_{2 n}, \mathfrak{Z}_{0}=\left(\begin{array}{cc}Z^{(n)} & 0 \\ 0 & W^{(n)}\end{array}\right) \in \mathfrak{H}_{2 n}$, we get

$$
\begin{aligned}
& \left(L^{k, l} E_{k}^{2 n}\right)(\mathfrak{Z}, s)=\sum_{M} \frac{1}{(2 \pi i)^{l}} \operatorname{det}\left(\mathfrak{C} \mathfrak{Z}_{0}+\mathfrak{D}\right)^{-k} \\
& \quad \times \operatorname{det}\left(\operatorname{Im}\left(M\left\langle\mathfrak{Z}_{0}\right\rangle\right)\right)^{s}\left\{\sum_{\nu=0}^{\frac{l}{2}} a(l, \nu, k, s) Q_{0}^{l-2 \nu}\left(P_{0}-P_{0}^{\prime}\right)^{\nu}\left(R_{0}-R_{0}^{\prime}\right)^{\nu}\right\}
\end{aligned}
$$

where $M=\left(\begin{array}{cc}* & * \\ \mathfrak{C}^{(2 n)} & \mathfrak{D}^{(2 n)}\end{array}\right)$ runs over a complete system of representatives of $P_{2 n, 0} \backslash \Gamma^{2 n}$,

$$
a(l, \nu, k, s)=\sum_{h=\nu}^{\left[\frac{l}{2}\right]}(-1)^{h-\nu}\left(\begin{array}{l}
h \\
\nu
\end{array}\right) b(l, h, k, s)
$$

$$
b(l, \nu, k, s)=(-1)^{l} \frac{(2 k-2+2 \nu)_{l-2 \nu}}{\nu !(l-2 \nu) !(k-1+\nu)_{l-\nu}} \frac{(-s)_{\nu}(k+s)_{l-\nu}}{(k)_{l}}
$$

$$
\left(0 \leq \nu \leq\left[\frac{l}{2}\right]\right)
$$

$$
\begin{array}{rlrl}
P_{0}=\left(\left(\mathfrak{C} \mathfrak{Z}_{0}+\mathfrak{D}\right)^{-1} \mathfrak{C}\right)\left[\left(\begin{array}{c}
{ }^{t} e_{1} \\
0
\end{array}\right)\right], & P_{0}^{\prime} & =\left(\frac{1}{2 i}\left(\operatorname{Im}\left(\mathfrak{Z}_{0}\right)\right)^{-1}\right)\left[\left(\begin{array}{c}
{ }^{t} e_{1} \\
0
\end{array}\right)\right], \\
Q_{0}=\left(\begin{array}{ll}
e_{1} & 0
\end{array}\right)\left(\left(\mathfrak{C} \mathfrak{Z}_{0}+\mathfrak{D}\right)^{-1} \mathfrak{C}\right)\left(\begin{array}{c}
0 \\
{ }^{t} e_{2}
\end{array}\right), & Q_{0}{ }^{\prime} & =\left(\begin{array}{ll}
e_{1} & 0
\end{array}\right)\left(\frac{1}{2 i}\left(\operatorname{Im}\left(\mathfrak{Z}_{0}\right)\right)^{-1}\right)\left(\begin{array}{c}
0 \\
{ }^{t} e_{2}
\end{array}\right) \\
& =0,
\end{array}
$$

$$
R_{0}=\left(\left(\mathfrak{C} \mathfrak{Z}_{0}+\mathfrak{D}\right)^{-1} \mathfrak{C}\right)\left[\left(\begin{array}{c}
0 \\
{ }^{t} e_{2}
\end{array}\right)\right], \quad R_{0}{ }^{\prime}=\left(\frac{1}{2 i}\left(\operatorname{Im}\left(\mathfrak{Z}_{0}\right)\right)^{-1}\right)\left[\left(\begin{array}{c}
0 \\
{ }^{t} e_{2}
\end{array}\right)\right]
$$

Now we put

$$
S_{0}=\left(\left(\mathfrak{C} \mathfrak{Z}_{0}+\mathfrak{D}\right)^{-1} \mathfrak{C}\right)\left[{ }^{t} x\right], \quad \delta=\operatorname{det}(\mathfrak{C} \mathfrak{Z}+\mathfrak{D}), \quad d^{*} \delta=\delta_{0}=\operatorname{det}\left(\mathfrak{C} \mathfrak{Z}_{0}+\mathfrak{D}\right),
$$




$$
S_{0}^{\prime}=\left(\frac{1}{2 i}\left(\operatorname{Im}\left(\mathfrak{Z}_{0}\right)\right)^{-1}\right)\left[{ }^{t} x\right], \quad \sigma=\operatorname{det}(\operatorname{Im}(\mathfrak{Z})), \quad d^{*} \sigma=\sigma_{0}=\operatorname{det}\left(\operatorname{Im}\left(\mathfrak{Z}_{0}\right)\right) .
$$

We note

$$
S_{0}=P_{0}+2 Q_{0}+R_{0}, \quad S_{0}{ }^{\prime}=P_{0}{ }^{\prime}+2 Q_{0}{ }^{\prime}+R_{0}{ }^{\prime}=P_{0}{ }^{\prime}+R_{0}{ }^{\prime}
$$

Since

$$
\operatorname{det}(\operatorname{Im}(M\langle\mathfrak{Z}\rangle))=|\operatorname{det}(\mathfrak{C} \mathfrak{Z}+\mathfrak{D})|^{-2} \operatorname{det}(\operatorname{Im}(\mathfrak{Z})), \quad \operatorname{Im}(\mathfrak{Z})=\frac{1}{2 i}(\mathfrak{Z}-\overline{\mathfrak{Z}}),
$$

we have only to prove Lemma 1 below so as to prove Proposition 1: we get

$$
\text { LEMMA 1. For } k, l \in \mathbb{Z}, k, l>0, s \in \mathbb{C} \text {, and } M=\left(\begin{array}{cc}
* & * \\
\mathfrak{C}^{(2 n)} & \mathfrak{D}^{(2 n)}
\end{array}\right) \in \Gamma^{2 n},
$$

$$
L^{k, l}\left(\delta^{-k-s} \sigma^{s}\right)=\frac{1}{(2 \pi i)^{l}} \delta_{0}^{-k-s} \sigma_{0}{ }^{s} \sum_{\nu=0}^{\left[\frac{l}{2}\right]} a(l, \nu, k, s) Q_{0}^{l-2 \nu}\left(P_{0}-P_{0}^{\prime}\right)^{\nu}\left(R_{0}-R_{0}^{\prime}\right)^{\nu}
$$

where notations are the same as those in Proposition 1.

In order to prove Lemma 1 inductively, we need the following Lemma:

Lemma 2. For $k, l \in \mathbb{Z}, k>0, l \geq 0$, we have

$$
L^{k, l}=\frac{1}{(2 \pi i) l(k+l-1)} L^{k, l-1} D_{0}+\frac{(k)_{l-2}(4-2 k-l)}{(2 \pi i)^{2} l(k)_{l}(2-k-l)_{2}} D_{\uparrow} D_{\downarrow} L^{k, l-2}
$$

Since $L^{k, l-1} D_{0} \neq D_{0} L^{k, l-1}(=0)$, we must consider what $L^{k, l-1} D_{0}\left(\delta^{-k-s} \sigma^{s}\right)$ is in a different way. We note $L^{k, l-1} D_{0}=d^{*} L_{*}^{k, l-1} D_{0}=d^{*} D_{0} L_{*}^{k, l-1}$. .

Lemma 3. For $k, l \in \mathbb{Z}, k>0, l \geq 2, s \in \mathbb{C}$, we obtain

$$
\begin{aligned}
L^{k, l-1} D_{0}\left(\delta^{-k-s} \sigma^{s}\right)= & d^{*} D_{0}\left(\left.L_{*}^{k, l-1}\right|_{Q^{\prime}=0}\right)\left(\delta^{-k-s} \sigma^{s}\right) \\
& +\frac{s}{(2 \pi i) k}\left(d^{*}\left(D_{0}^{2} \sigma\right)\right) \cdot L^{k+1, l-2}\left(\delta^{-k-s} \sigma^{s-1}\right)
\end{aligned}
$$

Proof of Lemma 2. We prove it for even $l$. The assertion for odd $l$ is proved in the same way. 
Let $l=2 m$. Then,

$$
\begin{aligned}
L^{k, 2 m}-\frac{1}{2 m(k+2 m-1)(2 \pi i)} L^{k, 2 m-1} D_{0} \\
=\frac{1}{(2 \pi i)^{l}(k)_{2 m}} d^{*}\left\{\sum _ { \nu = 1 } ^ { m - 1 } \left(\frac{1}{\nu !(2 m-2 \nu) !(2-k-2 m)_{\nu}}\right.\right. \\
\left.\left.\quad-\frac{1}{\nu ! 2 m(2 m-1-2 \nu) !(3-k-2 m)_{\nu}}\right)\left(D_{\uparrow} D_{\downarrow}\right)^{\nu} D_{0}^{2 m-2 \nu}\right\} \\
\quad+\frac{1}{(2 \pi i)^{l}(k)_{2 m} m !(2-k-2 m)_{m}} d^{*}\left(D_{\uparrow} D_{\downarrow}\right)^{m} .
\end{aligned}
$$

Here, we note

$$
\begin{aligned}
& \frac{1}{\nu !(2 m-2 \nu) !(2-k-2 m)_{\nu}}-\frac{1}{\nu ! 2 m(2 m-1-2 \nu) !(3-k-2 m)_{\nu}} \\
& \quad=\frac{4-2 k-2 m}{2 m(\nu-1) !(2 m-2 \nu) !(2-k-2 m)_{\nu+1}} .
\end{aligned}
$$

Therefore,

$$
\begin{aligned}
L^{k, 2 m}-\frac{1}{2 m(k+2 m-1)(2 \pi i)} L^{k, 2 m-1} D_{0} \\
=\frac{1}{(2 \pi i)^{2 m}(k)_{2 m}} D_{\uparrow} D_{\downarrow} d^{*} \sum_{\nu=1}^{m} \frac{4-2 k-2 m}{(\nu-1) ! 2 m(2 m-2 \nu) !(2-k-2 m)_{\nu+1}} \\
\quad \times\left(D_{\uparrow} D_{\downarrow}\right)^{\nu-1} D_{0}^{2 m-2 \nu} \\
=\frac{4-2 k-2 m}{(2 \pi i)^{2 m} 2 m(k)_{2 m}} D_{\uparrow} D_{\downarrow} d^{*} \sum_{\nu=0}^{m-1} \frac{1}{\nu !((2 m-2)-2 \nu) !(2-k-2 m)_{\nu+2}} \\
\quad \times\left(D_{\uparrow} D_{\downarrow}\right)^{\nu} D_{0}(2 m-2)-2 \nu \\
=\frac{(k)_{2 m-2}(4-2 k-2 m)}{(2 \pi i)^{2}(k)_{2 m} 2 m(2-k-2 m)_{2}} D_{\uparrow} D_{\downarrow} L^{k, 2 m-2} .
\end{aligned}
$$

We introduce some formulae before we prove Lemma 1 and Lemma 3.

When we replace $\mathfrak{Z}_{0}$ in $P_{0}, P_{0}{ }^{\prime}, \cdots, S_{0}, S_{0}{ }^{\prime}$ with $\mathfrak{Z}$, we write $P, P^{\prime}, \cdots, S, S^{\prime}$ for $P_{0}, P_{0}{ }^{\prime}, \cdots, S_{0}, S_{0}{ }^{\prime}$, respectively. We note $d^{*} P=P_{0}, d^{*} P^{\prime}=P_{0}{ }^{\prime}, \cdots, d^{*} S=S_{0}{ }^{\prime}$ and $d^{*} S^{\prime}=S_{0}{ }^{\prime}$. Then we get the following table: 


\begin{tabular}{c|c|c|c|c|c}
\hline & $\delta$ & $S$ & $P$ & $Q$ & $R$ \\
\hline$D$ & $\delta S$ & $-S^{2}$ & $-(P+Q)^{2}$ & $-(P+Q)(R+Q)$ & $-(R+Q)^{2}$ \\
$D_{\uparrow}$ & $\delta P$ & $-(P+Q)^{2}$ & $-P^{2}$ & $-P Q$ & $-Q^{2}$ \\
$D_{0}$ & $2 \delta Q$ & $-2(P+Q)(R+Q)$ & $-2 P Q$ & $-P R-Q^{2}$ & $-2 R Q$ \\
$D_{\downarrow}$ & $\delta R$ & $-(R+Q)^{2}$ & $-Q^{2}$ & $-R Q$ & $-R^{2}$ \\
\hline
\end{tabular}

Moreover, we get

$$
\begin{gathered}
D_{\uparrow} D_{0} \delta=D_{0} D_{\uparrow} \delta=0, \quad D_{\downarrow} D_{0} \delta=D_{0} D_{\downarrow} \delta=0, \quad D_{0}^{3} \delta=0, \\
D_{0}^{2} \delta=D_{0}(2 \delta Q)=-2 \delta\left(P R-Q^{2}\right), \quad D_{\uparrow} D_{\downarrow} \delta=\delta\left(P R-Q^{2}\right) .
\end{gathered}
$$

If we replace $\delta, S, \cdots, R$ with $\sigma, S^{\prime}, \cdots, R^{\prime}$ respectively, we obtain formulae for $\sigma, S^{\prime}, \cdots, R^{\prime}$ in the same way. We also get tables for $\delta_{0}$ and for $\sigma_{0}$ if we only note $D_{0} d^{*}=0$ and $Q_{0}{ }^{\prime}=0$.

Proof of Lemma 3. We recall $L^{k, l-1} D_{0}\left(\delta^{-k-s} \sigma^{s}\right)=d^{*} D_{0} L_{*}^{k, l-1}\left(\delta^{-k-s} \sigma^{s}\right)$. Then we have

$$
L_{*}^{k, l-1}\left(\delta^{-k-s} \sigma^{s}\right)=\delta^{-k-s} \sigma^{s} H,
$$

where $H$ is a polynomial in $P, Q, R, P^{\prime}, Q^{\prime}$ and $R^{\prime}$. Moreover, we have an expansion

$$
L_{*}^{k, l-1}\left(\delta^{-k-s} \sigma^{s}\right)=\delta^{-k-s} \sigma^{s} \sum_{r=0}^{h} H_{r} Q^{\prime r}
$$

where $H_{r}$ is a polynomial in $P, Q, R, P^{\prime}$ and $R^{\prime}$, and $h<\infty$. By the table above and so on, we obtain

$$
d^{*} D_{0} L_{*}^{k, l-1}\left(\delta^{-k-s} \sigma^{s}\right)=d^{*} D_{0}\left(\delta^{-k-s} \sigma^{s}\left(H_{0}+H_{1} Q^{\prime}\right)\right)
$$

and

$$
d^{*} D_{0}\left(\delta^{-k-s} \sigma^{s} H_{0}\right)=d^{*} D_{0}\left(\left.L_{*}^{k, l-1}\right|_{Q^{\prime}=0}\right)\left(\delta^{-k-s} \sigma^{s}\right)
$$

On the other hand, $d^{*} D_{0}\left(\delta^{-k-s} \sigma^{s} H_{1} Q^{\prime}\right)$ is given in the following way:

We consider $\delta^{-k-s} \sigma^{s}$ a composite function of $\delta^{-k-s}$ and $\sigma^{s}$. Once a factor $D_{0}$ of $\left(D_{\uparrow} D_{\downarrow}\right)^{\nu} D_{0}{ }^{(l-1)-2 \nu}\left(0 \leq \nu \leq\left[\frac{l-1}{2}\right]\right)$ in $L_{*}^{k, l-1}$ acts on $\sigma^{s}$, nothing acts on $D_{0} \sigma\left(=2 \sigma Q^{\prime}\right)$ which comes from $D_{0}\left(\sigma^{s}\right)=s \sigma^{s-1}\left(D_{0} \sigma\right)$. That is, 


$$
\begin{aligned}
& d^{*} D_{0}\left(\delta^{-k-s} \sigma^{s} H_{1} Q^{\prime}\right) d^{*} D_{0}\left\{\left(\frac{1}{(2 \pi i)^{l-1}(k)_{l-1}} \sum_{\nu=0}^{\left[\frac{l}{2}\right]-1} \frac{1}{\nu !(l-1-2 \nu) !(2-k-(l-1))_{\nu}}\right.\right. \\
&\left.\left.\times\left(D_{\uparrow} D_{\downarrow}\right)^{\nu} D_{0}^{l-2-2 \nu}\left(\delta^{-k-s} \sigma^{s-1}\right)\right)\left(\begin{array}{c}
l-1-2 \nu \\
1
\end{array}\right) s\left(D_{0} \sigma\right)\right\} \\
&= \frac{1}{(2 \pi i)^{l-1}(k)_{l-1}} d^{*} \sum_{\nu=0}^{\left[\frac{l-2}{2}\right]} \frac{1}{\nu !(l-1-2 \nu) !(2-k-(l-1))_{\nu}} \\
&=\left\{\left(D_{\uparrow} D_{\downarrow}\right)^{\nu} D_{0}^{l-2-2 \nu}\left(\delta^{-k-s} \sigma^{s-1}\right)\right\}\left(\begin{array}{c}
l-1-2 \nu \\
1
\end{array}\right) s\left(d^{*}\left(D_{0}^{2} \sigma\right)\right) \\
& \frac{1}{(2 \pi i)^{l-2}(k+1)_{l-2}} d^{*} \sum_{\nu=0}^{\left[\frac{l-2}{2}\right]} \frac{1}{\nu !(l-2-2 \nu) !(2-(k+1)-(l-2))_{\nu}} \\
&\left.\quad \times\left(D_{\uparrow} D_{\downarrow}\right)^{\nu} D_{0}^{l-2-2 \nu}\left(\delta^{-k-s} \sigma^{s-1}\right)\right\} \frac{s}{(2 \pi i) k}\left(d^{*}\left(D_{0}^{2} \sigma\right)\right) \\
& \frac{s \quad\left(d^{*}\left(D_{0}^{2} \sigma\right)\right) \cdot L^{k+1, l-2}\left(\delta^{-k-s} \sigma^{s-1}\right) .}{(2 \pi i) k}
\end{aligned}
$$

REMARK. For odd $l, L_{*}^{k, l-1}$ has a term $\left(D_{\uparrow} D_{\downarrow}\right)^{\frac{l-1}{2}}$, but $\left(D_{\uparrow} D_{\downarrow}\right)^{\frac{l-1}{2}}$ has no relation to $d^{*} D_{0}\left(\delta^{-k-s} \sigma^{s} H_{1} Q^{\prime}\right)$ because $d^{*} D_{0}\left(D_{\uparrow} D_{\downarrow}\right)^{\frac{l-1}{2}}=\left(D_{\uparrow} D_{\downarrow}\right)^{\frac{l-1}{2}} d^{*} D_{0}$.

Proof of Lemma 1. We use induction on $l$. For $l=1$ and for $l=2$, Lemma 1 holds.

Let $l>2$. By Lemma 2, we have

$$
\begin{aligned}
L^{k, l}\left(\delta^{-k-s} \sigma^{s}\right) \\
=\frac{1}{(2 \pi i) l(k+l-1)} L^{k, l-1} D_{0}\left(\delta^{-k-s} \sigma^{s}\right) \\
\quad+\frac{(k)_{l-2}(4-2 k-l)}{(2 \pi i)^{2}(k)_{l} l(2-k-l)_{2}} D_{\uparrow} D_{\downarrow} L^{k, l-2}\left(\delta^{-k-s} \sigma^{s}\right)
\end{aligned}
$$


by Lemma 3,

$$
\begin{aligned}
= & \frac{1}{(2 \pi i) l(k+l-1)} d^{*} D_{0}\left(\left.L_{*}^{k, l-1}\right|_{Q^{\prime}=0}\right)\left(\delta^{-k-s} \sigma^{s}\right) \\
& +\frac{s}{(2 \pi i)^{2} l(k+l-1) k}\left(d^{*}\left(D_{0}^{2} \sigma\right)\right) L^{k+1, l-2}\left(\delta^{-k-s} \sigma^{s-1}\right) \\
& +\frac{(k)_{l-2}(4-2 k-l)}{(2 \pi i)^{2}(k)_{l} l(2-k-l)_{2}} D_{\uparrow} D_{\downarrow} L^{k, l-2}\left(\delta^{-k-s} \sigma^{s}\right)
\end{aligned}
$$

since the induction hypothesis is valid for $\left(\left.L_{*}^{k, l-1}\right|_{Q^{\prime}=0}\right)\left(\delta^{-k-s} \sigma^{s}\right)$

$$
\begin{aligned}
& =\frac{1}{(2 \pi i)^{l} l(k+l-1)} \\
& \quad \times d^{*} D_{0}\left(\sum_{\nu=0}^{\left[\frac{l-1}{2}\right]} \delta^{-k-s} \sigma^{s} a(l-1, \nu, k, s) Q^{l-1-2 \nu}\left(P-P^{\prime}\right)^{\nu}\left(R-R^{\prime}\right)^{\nu}\right) \\
& +\frac{-2 s}{(2 \pi i)^{l} l(k+l-1) k} \\
& \times\left\{\begin{array}{l}
{\left[\frac{l}{2}\right]-1} \\
\sum_{\nu=0} \delta_{0}^{-k-s} \sigma_{0}^{s} a(l-2, \nu, k+1, s-1) Q_{0}^{l-2-2 \nu}\left(P_{0}-P_{0}^{\prime}\right)^{\nu}\left(R_{0}-R_{0}^{\prime}\right)^{\nu}
\end{array}\right\} P_{0}^{\prime} R_{0}^{\prime} \\
& +\frac{(k)_{l-2}(4-2 k-l)}{(2 \pi i)^{l}(k)_{l} l(2-k-l)_{2}} \\
& \quad \times D_{\uparrow} D_{\downarrow}\left(\sum_{\nu=0}^{\left[\frac{l}{2}\right]-1} \delta_{0}^{-k-s} \sigma_{0}^{s} a(l-2, \nu, k, s) Q_{0}^{l-2-2 \nu}\left(P_{0}-P_{0}^{\prime}\right)^{\nu}\left(R_{0}-R_{0}^{\prime}\right)^{\nu}\right) .
\end{aligned}
$$

On the other hand, we have

$$
\begin{aligned}
& d^{*} D_{0}\left(\delta^{-k-s} \sigma^{s} Q^{l-1-2 \nu}\left(P-P^{\prime}\right)^{\nu}\left(R-R^{\prime}\right)^{\nu}\right) \\
& =-(2 k+2 s+l-1-2 \nu) \delta_{0}{ }^{-k-s} \sigma_{0}^{s} Q_{0}{ }^{l-2 \nu}\left(P_{0}-P_{0}^{\prime}\right)^{\nu}\left(R_{0}-R_{0}^{\prime}\right)^{\nu} \\
& \quad-4 \nu \delta_{0}^{-k-s} \sigma_{0}^{s} Q_{0}^{l-2 \nu} P_{0} R_{0}\left(P_{0}-P_{0}^{\prime}\right)^{\nu-1}\left(R_{0}-R_{0}^{\prime}\right)^{\nu-1} \\
& \quad+2 \nu \delta_{0}{ }^{-k-s} \sigma_{0}{ }^{s} Q_{0}{ }^{l-2 \nu}\left(P_{0} R_{0}^{\prime}+P_{0}^{\prime} R_{0}\right)\left(P_{0}-P_{0}^{\prime}\right)^{\nu-1}\left(R_{0}-R_{0}^{\prime}\right)^{\nu-1} \\
& \quad-(l-1-2 \nu) \delta_{0}{ }^{-k-s} \sigma_{0}^{s} Q_{0}^{l-2-2 \nu} P_{0} R_{0}\left(P_{0}-P_{0}^{\prime}\right)^{\nu}\left(R_{0}-R_{0}^{\prime}\right)^{\nu} \\
& \quad\left(0 \leq \nu \leq\left[\frac{l-1}{2}\right]\right),
\end{aligned}
$$


and

$$
\begin{aligned}
& D_{\uparrow} D_{\downarrow}\left(\delta_{0}^{-k-s} \sigma_{0}^{s} Q_{0}^{l-2-2 \nu}\left(P_{0}-P_{0}^{\prime}\right)^{\nu}\left(R_{0}-R_{0}^{\prime}\right)^{\nu}\right) \\
& =\nu^{2} \delta_{0}{ }^{k-s} \sigma_{0}{ }^{s} Q_{0}{ }^{l-2 \nu+2}\left(P_{0}-P_{0}^{\prime}\right)^{\nu-1}\left(R_{0}-R_{0}^{\prime}\right)^{\nu-1} \\
& +(k+s+l-2-\nu) \delta_{0}{ }^{-k-s} \sigma_{0}{ }^{s} Q_{0}{ }^{l-2 \nu}\left(P_{0}-P_{0}^{\prime}\right)^{\nu}\left(R_{0}-R_{0}^{\prime}\right)^{\nu} \\
& +\nu(2 k+2 s+2 l-2 \nu-3) \delta_{0}{ }^{-k-s} \sigma_{0}{ }^{s} Q_{0}{ }^{l-2 \nu} P_{0} R_{0}\left(P_{0}-P_{0}^{\prime}\right)^{\nu-1}\left(R_{0}-R_{0}^{\prime}\right)^{\nu-1} \\
& -\nu(k+2 s+l-2 \nu-1) \delta_{0}{ }^{-k-s} \sigma_{0}{ }^{s} Q_{0}{ }^{l-2 \nu}\left(P_{0} R_{0}^{\prime}+P_{0}^{\prime} R_{0}\right)\left(P_{0}-P_{0}^{\prime}\right)^{\nu-1} \\
& \times\left(R_{0}-R_{0}^{\prime}\right)^{\nu-1} \\
& +\nu(2 s-2 \nu+1) \delta_{0}{ }^{-k-s} \sigma_{0} s Q_{0}{ }^{l-2 \nu} P_{0}^{\prime} R_{0}^{\prime}\left(P_{0}-P_{0}^{\prime}\right)^{\nu-1}\left(R_{0}-R_{0}^{\prime}\right)^{\nu-1} \\
& +(k+s+l-2-\nu)^{2} \delta_{0}{ }^{-k-s} \sigma_{0}{ }^{s} Q_{0}{ }^{l-2 \nu-2} P_{0} R_{0}\left(P_{0}-P_{0}^{\prime}\right)^{\nu}\left(R_{0}-R_{0}^{\prime}\right)^{\nu} \\
& -(k+s+l-2-\nu)(s-\nu) \delta_{0}^{-k-s} \sigma_{0}^{s} Q_{0}^{l-2 \nu-2}\left(P_{0} R_{0}^{\prime}+P_{0}^{\prime} R_{0}\right)\left(P_{0}-P_{0}^{\prime}\right)^{\nu} \\
& \times\left(R_{0}-R_{0}^{\prime}\right)^{\nu} \\
& +(s-\nu)^{2} \delta_{0}^{-k-s} \sigma_{0}^{s} Q_{0}^{l-2 \nu-2} P_{0}^{\prime} R_{0}^{\prime}\left(P_{0}-P_{0}^{\prime}\right)^{\nu}\left(R_{0}-R_{0}^{\prime}\right)^{\nu} \\
& \left(0 \leq \nu \leq\left[\frac{l}{2}\right]-1\right) \text {. }
\end{aligned}
$$

Thus we have only to prove the following equations:

(1) coefficient of $Q_{0}{ }^{l}$

$$
\begin{gathered}
a(l, 0, k, s)=\frac{1}{l(k+l-1)}\{-(2 k+2 s+l-1) a(l-1,0, k, s)\} \\
+\frac{(k)_{l-2}(4-2 k-l)}{(k)_{l} l(2-k-l)_{2}}\{(k+s+l-2) a(l-2,0, k, s) \\
+a(l-2,1, k, s)\}
\end{gathered}
$$

(2) coefficient of ${Q_{0}}^{l-2 \nu} P_{0} R_{0}\left(P_{0}-P_{0}^{\prime}\right)^{\nu-1}\left(R_{0}-R_{0}^{\prime}\right)^{\nu-1} \quad\left(1 \leq \nu \leq\left[\frac{l}{2}\right]-2\right)$

$$
\begin{aligned}
& a(l, \nu, k, s)=\frac{1}{l(k+l-1)}\{-(2 k+2 s+l-1+2 \nu) a(l-1, \nu, k, s) \\
&-(l+1-2 \nu) a(l-1, \nu-1, k, s)\} \\
&+\frac{(k)_{l-2}(4-2 k-l)}{(k)_{l} l(2-k-l)_{2}}\left\{(\nu+1)^{2} a(l-2, \nu+1, k, s)\right. \\
&+(k+s+l-2-\nu) a(l-2, \nu, k, s) \\
&+\nu(2 k+2 s+2 l-2 \nu-3) a(l-2, \nu, k, s) \\
&\left.+(k+s+l-1-\nu)^{2} a(l-2, \nu-1, k, s)\right\}
\end{aligned}
$$


(3) coefficient of $-Q_{0}^{l-2 \nu}\left(P_{0} R_{0}^{\prime}+P_{0}^{\prime} R_{0}\right)\left(P_{0}-P_{0}^{\prime}\right)^{\nu-1}\left(R_{0}-R_{0}^{\prime}\right)^{\nu-1}$ $\left(1 \leq \nu \leq\left[\frac{l}{2}\right]-2\right)$

$$
\begin{aligned}
a(l, \nu, k, s)= & \frac{1}{l(k+l-1)}\{-(2 k+2 s+l-1) a(l-1, \nu, k, s)\} \\
& +\frac{(k)_{l-2}(4-2 k-l)}{(k)_{l} l(2-k-l)_{2}}\left\{(\nu+1)^{2} a(l-2, \nu+1, k, s)\right. \\
& \quad+(k+s+l-2-\nu) a(l-2, \nu, k, s) \\
& +\nu(k+2 s+l-2 \nu-1) a(l-2, \nu, k, s) \\
& +(k+s+l-1-\nu)(s-\nu+1) a(l-2, \nu-1, k, s)\}
\end{aligned}
$$

(4) coefficient of ${Q_{0}}^{l-2 \nu} P_{0}^{\prime} R_{0}^{\prime}\left(P_{0}-P_{0}^{\prime}\right)^{\nu-1}\left(R_{0}-R_{0}^{\prime}\right)^{\nu-1} \quad\left(1 \leq \nu \leq\left[\frac{l}{2}\right]-2\right)$

$$
\begin{aligned}
a(l, \nu, k, s)= & \frac{1}{l(k+l-1)}\{-(2 k+2 s+l-1-2 \nu) a(l-1, \nu, k, s) \\
& \left.\quad-\frac{2 s}{k} a(l-2, \nu-1, k+1, s-1)\right\} \\
& +\frac{(k)_{l-2}(4-2 k-l)}{(k)_{l} l(2-k-l)_{2}}\left\{(\nu+1)^{2} a(l-2, \nu+1, k, s)\right. \\
& +(k+s+l-2-\nu) a(l-2, \nu, k, s) \\
& \left.+\nu(2 s-2 \nu+1) a(l-2, \nu, k, s)+(s-\nu+1)^{2} a(l-2, \nu-1, k, s)\right\}
\end{aligned}
$$

(5) coefficient of $Q_{0}^{l-2 \nu} P_{0} R_{0}\left(P_{0}-P_{0}^{\prime}\right)^{\nu-1}\left(R_{0}-R_{0}^{\prime}\right)^{\nu-1} \quad\left(\nu=\left[\frac{l}{2}\right]-1\right)$

$$
\begin{aligned}
a(l, \nu, k, s)=\frac{1}{l(k+l-1)}\{-(2 k+2 s+l-1+2 \nu) a(l-1, \nu, k, s) \\
-(l+1-2 \nu) a(l-1, \nu-1, k, s)\} \\
+\frac{(k)_{l-2}(4-2 k-l)}{(k)_{l} l(2-k-l)_{2}}\{(k+s+l-2-\nu) a(l-2, \nu, k, s) \\
+\nu(2 k+2 s+2 l-2 \nu-3) a(l-2, \nu, k, s) \\
\left.+(k+s+l-1-\nu)^{2} a(l-2, \nu-1, k, s)\right\} .
\end{aligned}
$$

(6) coefficient of $-Q_{0}{ }^{l-2 \nu}\left(P_{0} R_{0}^{\prime}+P_{0}^{\prime} R_{0}\right)\left(P_{0}-P_{0}^{\prime}\right)^{\nu-1}\left(R_{0}-R_{0}^{\prime}\right)^{\nu-1}$ $\left(\nu=\left[\frac{l}{2}\right]-1\right)$ 


$$
\begin{aligned}
a(l, \nu, k, s)= & \frac{1}{l(k+l-1)}\{-(2 k+2 s+l-1) a(l-1, \nu, k, s)\} \\
+ & \frac{(k)_{l-2}(4-2 k-l)}{(k)_{l} l(2-k-l)_{2}}\{(k+s+l-2-\nu) a(l-2, \nu, k, s) \\
& +\nu(k+2 s+l-2 \nu-1) a(l-2, \nu, k, s) \\
& +(k+s+l-1-\nu)(s-\nu+1) a(l-2, \nu-1, k, s)\} .
\end{aligned}
$$

(7) coefficient of $Q_{0}^{l-2 \nu} P_{0}^{\prime} R_{0}^{\prime}\left(P_{0}-P_{0}^{\prime}\right)^{\nu-1}\left(R_{0}-R_{0}^{\prime}\right)^{\nu-1} \quad\left(\nu=\left[\frac{l}{2}\right]-1\right)$

$$
\begin{aligned}
& a(l, \nu, k, s)=\frac{1}{l(k+l-1)}\{-(2 k+2 s+l-1-2 \nu) a(l-1, \nu, k, s) \\
& \left.-\frac{2 s}{k} a(l-2, \nu-1, k+1, s-1)\right\} \\
& +\frac{(k)_{l-2}(4-2 k-l)}{(k)_{l} l(2-k-l)_{2}}\{(k+s+l-2-\nu) a(l-2, \nu, k, s) \\
& +\nu(2 s-2 \nu+1) a(l-2, \nu, k, s) \\
& \left.+(s-\nu+1)^{2} a(l-2, \nu-1, k, s)\right\} \text {. }
\end{aligned}
$$

For even $l$, we put $l=2 m$.

(8) coefficient of $P_{0} R_{0}\left(P_{0}-P_{0}^{\prime}\right)^{m-1}\left(R_{0}-R_{0}^{\prime}\right)^{m-1}$

$$
\begin{aligned}
a(l, m, k, s)= & \frac{1}{l(k+l-1)}\{-a(l-1, m-1, k, s)\} \\
& +\frac{(k)_{l-2}(4-2 k-l)}{(k)_{l} l(2-k-l)_{2}}\left\{(k+s+m-1)^{2} a(l-2, m-1, k, s)\right\} .
\end{aligned}
$$

(9) coefficient of $-\left(P_{0} R_{0}^{\prime}+P_{0}^{\prime} R_{0}\right)\left(P_{0}-P_{0}^{\prime}\right)^{m-1}\left(R_{0}-R_{0}^{\prime}\right)^{m-1}$

$$
\begin{aligned}
& a(l, m, k, s) \\
& \quad=\frac{(k)_{l-2}(4-2 k-l)}{(k)_{l} l(2-k-l)_{2}}\{(k+s+m-1)(s-m+1) a(l-2, m-1, k, s)\} .
\end{aligned}
$$

(10) coefficient of $P_{0}^{\prime} R_{0}^{\prime}\left(P_{0}-P_{0}^{\prime}\right)^{m-1}\left(R_{0}-R_{0}^{\prime}\right)^{m-1}$

$$
\begin{aligned}
a(l, m, k, s)= & \frac{1}{l(k+l-1)}\left\{-\frac{2 s}{k} a(l-2, m-1, k+1, s-1)\right\} \\
& +\frac{(k)_{l-2}(4-2 k-l)}{(k)_{l} l(2-k-l)_{2}}\left\{(s-m+1)^{2} a(l-2, m-1, k, s)\right\} .
\end{aligned}
$$


For odd $l$, we put $l=2 m+1$.

$\left(8^{\prime}\right)$. coefficient of $Q_{0} P_{0} R_{0}\left(P_{0}-P_{0}^{\prime}\right)^{m-1}\left(R_{0}-R_{0}^{\prime}\right)^{m-1}$

$$
\begin{aligned}
a(l, m, k, s)= & \frac{1}{l(k+l-1)}\{-(2 k+2 s+4 m) a(l-1, m, k, s) \\
& -2 a(l-1, m-1, k, s)\} \\
+ & \frac{(k)_{l-2}(4-2 k-l)}{(k)_{l} l(2-k-l)_{2}}\left\{(k+s+m)^{2} a(l-2, m-1, k, s)\right\} .
\end{aligned}
$$

$\left(9^{\prime}\right) \quad$ coefficient of $-Q_{0}\left(P_{0} R_{0}^{\prime}+P_{0}^{\prime} R_{0}\right)\left(P_{0}-P_{0}^{\prime}\right)^{m-1}\left(R_{0}-R_{0}^{\prime}\right)^{m-1}$

$$
\begin{aligned}
a(l, m, k, s)= & \frac{1}{l(k+l-1)}\{-(2 k+2 s+2 m) a(l-1, m, k, s)\} \\
& +\frac{(k)_{l-2}(4-2 k-l)}{(k)_{l} l(2-k-l)_{2}}\{(k+s+m)(s-m+1) a(l-2, m-1, k, s)\}
\end{aligned}
$$

$\left(10^{\prime}\right)$ coefficient of $Q_{0} P_{0}^{\prime} R_{0}^{\prime}\left(P_{0}-P_{0}^{\prime}\right)^{m-1}\left(R_{0}-R_{0}^{\prime}\right)^{m-1}$

$$
\begin{aligned}
a(l, m, k, s)= & \frac{1}{l(k+l-1)}\{-(2 k+2 s) a(l-1, m, k, s) \\
& \left.-\frac{2 s}{k} a(l-2, m-1, k+1, s-1)\right\} \\
& +\frac{(k)_{l-2}(4-2 k-l)}{(k)_{l} l(2-k-l)_{2}}\left\{(s-m+1)^{2} a(l-2, m-1, k, s)\right\} .
\end{aligned}
$$

By properties of $a(l, \nu, k, s)$, it is easy to prove (5), (6), (7), (8), (9), (10), ( $\left.8^{\prime}\right)$, $\left(9^{\prime}\right),(10)$. Therefore, we have only to prove $(1),(2),(3),(4)$. But we only prove $(2)$ for even $l$, here. The assertions of (1), (3), (4) and of (2) for odd $l$ are proved in the same way.

Let $l=2 m$. Then, by (2.4), the right-hand side of $(2)$ is:

$$
\begin{array}{r}
\frac{1}{2 m(k+2 m-1)}\left\{-(2 k+2 s+2 m-1+2 \nu) \sum_{h=\nu}^{m-1}(-1)^{h-\nu}\left(\begin{array}{l}
h \\
\nu
\end{array}\right) b(2 m-1, h, k, s)\right. \\
\left.-(2 m+1-2 \nu) \sum_{h=\nu-1}^{m-1}(-1)^{h+1-\nu}\left(\begin{array}{c}
h \\
\nu-1
\end{array}\right) b(2 m-1, h, k, s)\right\} \\
-\frac{(k)_{2 m-2}(2 k+2 m-4)}{(k)_{2 m} 2 m(k+2 m-3)_{2}}\left\{(\nu+1)^{2} \sum_{h=\nu+1}^{m-1}(-1)^{h-\nu+1}\left(\begin{array}{c}
h \\
\nu+1
\end{array}\right) b(2 m-2, h, k, s)\right.
\end{array}
$$




$$
\begin{gathered}
+(k+s+2 m-\nu-1)^{2} \sum_{h=\nu-1}^{m-1}(-1)^{h+1-\nu}\left(\begin{array}{c}
h \\
\nu-1
\end{array}\right) b(2 m-2, h, k, s) \\
+((k+s+2 m-\nu-2)+\nu(2 k+2 s+4 m-2 \nu-3)) \\
\left.\times \sum_{h=\nu}^{m-1}(-1)^{h-\nu}\left(\begin{array}{c}
h \\
\nu
\end{array}\right) b(2 m-2, h, k, s)\right\}
\end{gathered}
$$

$$
\begin{aligned}
=\frac{1}{2 m(k+2 m-1)}\left\{\sum _ { h = \nu - 1 } ^ { m - 1 } ( - 1 ) ^ { h - \nu } \left(\left(\begin{array}{c}
h \\
\nu-1
\end{array}\right)(2 k+2 s+4 m)\right.\right. \\
\left.\left.\quad-\left(\begin{array}{c}
h+1 \\
\nu
\end{array}\right)(2 k+2 s+2 m+2 \nu-1)\right) b(2 m-1, h, k, s)\right\} \\
+\frac{(k)_{2 m-2}(2 k+2 m-4)}{(k)_{2 m} 2 m(k+2 m-3)_{2}}\left\{-(k+s+2 m-\nu-1)^{2} b(2 m-2, \nu-1, k, s)\right. \\
+\sum_{h=\nu}^{m-1}(-1)^{h-\nu}\left(\left(\begin{array}{c}
h \\
\nu-1
\end{array}\right)(k+s+2 m-\nu-1)(k+s+2 m-h-2)\right. \\
\left.\left.-\left(\begin{array}{c}
h \\
\nu
\end{array}\right)(\nu+1)(k+s+2 m-h-2)\right) b(2 m-2, h, k, s)\right\} .
\end{aligned}
$$

We note

$$
\begin{gathered}
\left(\begin{array}{c}
h \\
\nu-1
\end{array}\right)(2 k+2 s+4 m)-\left(\begin{array}{c}
h+1 \\
\nu
\end{array}\right)(2 k+2 s+2 m+2 \nu-1) \\
=\left(\begin{array}{c}
h+1 \\
\nu
\end{array}\right)(2 m-2 h-1)-2\left(\begin{array}{l}
h \\
\nu
\end{array}\right)(k+s+2 m-h-1)
\end{gathered}
$$

$$
\begin{aligned}
& \left(\begin{array}{c}
h \\
\nu-1
\end{array}\right)(k+s+2 m-\nu-1)-\left(\begin{array}{l}
h \\
\nu
\end{array}\right)(\nu+1) \\
& =\left(\begin{array}{c}
h \\
\nu-1
\end{array}\right)(k+s+2 m-h-1)+\left(\begin{array}{c}
h \\
\nu-1
\end{array}\right)(h-\nu)-\left(\begin{array}{l}
h \\
\nu
\end{array}\right)(\nu+1) \\
& =\left(\begin{array}{c}
h \\
\nu-1
\end{array}\right)(k+s+2 m-h-1)-\left(\begin{array}{c}
h+1 \\
\nu
\end{array}\right)
\end{aligned}
$$

and

(2.9) $(k+s+2 m-\nu-1)^{2}=(k+s+2 m-\nu-1)_{2}-(k+s+2 m-\nu-1)$. 
By (2.7), (2.8), (2.9), the formula (2.6) is:

$$
\begin{array}{r}
\frac{1}{2 m(k+2 m-1)}\left\{\sum_{h=\nu-1}^{m-1}(-1)^{h-\nu}\left(\begin{array}{c}
h+1 \\
\nu
\end{array}\right)(2 m-2 h-1) b(2 m-1, h, k, s)\right. \\
\left.-\sum_{h=\nu}^{m-1}(-1)^{h-\nu}\left(\begin{array}{c}
h \\
\nu
\end{array}\right) 2(k+s+2 m-h-1) b(2 m-1, h, k, s)\right\} \\
+\frac{(k)_{2 m-2}(2 k+2 m-4)}{(k)_{2 m} 2 m(k+2 m-3)_{2}}\left\{\begin{array}{c}
\sum_{h=\nu-1}^{m-1}(-1)^{h-\nu}\left(\left(\begin{array}{c}
h \\
\nu-1
\end{array}\right)(k+s+2 m-h-2)_{2}\right. \\
\left.\left.-\left(\begin{array}{c}
h+1 \\
\nu
\end{array}\right)(k+s+2 m-h-2)\right) b(2 m-2, h, k, s)\right\}
\end{array}\right.
\end{array}
$$

by $(2.5)$

$$
\begin{aligned}
=-\sum_{h=\nu-1}^{m-1}(-1)^{h-\nu}\left(\begin{array}{c}
h+1 \\
\nu
\end{array}\right) \frac{(2 k+2 h-2)_{2 m-2 h-1}}{2 m(h) !(2 m-2 h-2) !(k+h-1)_{2 m-h-1}} \\
\quad \times \frac{(-s)_{h}(k+s)_{2 m-h-1}}{(k)_{2 m}} \\
+\sum_{h=\nu}^{m-1}(-1)^{h-\nu}\left(\begin{array}{l}
h \\
\nu
\end{array}\right) \frac{(2 k+2 h-2)_{2 m-2 h-1}}{m(h) !(2 m-2 h-1) !(k+h-1)_{2 m-h-1}} \\
\times \frac{(-s)_{h}(k+s)_{2 m-h}}{(k)_{2 m}}
\end{aligned}
$$

$$
\begin{aligned}
+\sum_{h=\nu-1}^{m-1}(-1)^{h-\nu}\left(\begin{array}{c}
h \\
\nu-1
\end{array}\right) \frac{(2 k+2 h-2)_{2 m-2 h-1}}{2 m(h) !(2 m-2 h-2) !(k+h-1)_{2 m-h}} \\
\times \frac{(-s)_{h}(k+s)_{2 m-h}}{(k)_{2 m}} \\
-\sum_{h=\nu-1}^{m-1}(-1)^{h-\nu}\left(\begin{array}{c}
h+1 \\
\nu
\end{array}\right) \frac{(2 k+2 h-2)_{2 m-2 h-1}}{2 m(h) !(2 m-2 h-2) !(k+h-1)_{2 m-h}} \\
\times \frac{(-s)_{h}(k+s)_{2 m-h-1}}{(k)_{2 m}} .
\end{aligned}
$$


We also note

$$
\begin{aligned}
& \frac{1}{m(h) !(2 m-2 h-1) !(k+h-1)_{2 m-h-1}} \\
& =\frac{2 k+2 m-3}{h !(2 m-2 h) !(k+h-1)_{2 m-h}}+\frac{1}{2 m(h) !(2 m-2 h-2) !(k+h-1)_{2 m-h}} \\
& \quad-\frac{2 k+2 h-3}{m(h-1) !(2 m-2 h) !(k+h-1)_{2 m-h}} .
\end{aligned}
$$

If we add the first term of (2.10) to the fourth term of (2.10) and if we transform the second term of (2.10) according to (2.11), we get:

$$
\begin{aligned}
& \sum_{h=\nu}^{m-1}(-1)^{h-\nu}\left(\begin{array}{l}
h \\
\nu
\end{array}\right) \frac{(2 k+2 h-2)_{2 m-2 h}}{h !(2 m-2 h) !(k+h-1)_{2 m-h}} \frac{(-s)_{h}(k+s)_{2 m-h}}{(k)_{2 m}} \\
& +\sum_{h=\nu}^{m-1}(-1)^{h-\nu}\left(\begin{array}{l}
h \\
\nu
\end{array}\right) \frac{(2 k+2 h-2)_{2 m-2 h-1}}{2 m(h) !(2 m-2 h-2) !(k+h-1)_{2 m-h}} \frac{(-s)_{h}(k+s)_{2 m-h}}{(k)_{2 m}} \\
& -\sum_{h=\nu}^{m-1}(-1)^{h-\nu}\left(\begin{array}{l}
h \\
\nu
\end{array}\right) \frac{(2 k+2 h-3)_{2 m-2 h}}{m(h-1) !(2 m-2 h) !(k+h-1)_{2 m-h}} \\
& \times \frac{(-s)_{h}(k+s)_{2 m-h}}{(k)_{2 m}} \\
& +\sum_{h=\nu-1}^{m-1}(-1)^{h-\nu}\left(\begin{array}{c}
h \\
\nu-1
\end{array}\right) \frac{(2 k+2 h-2)_{2 m-2 h-1}}{2 m(h) !(2 m-2 h-2) !(k+h-1)_{2 m-h}} \\
& \times \frac{(-s)_{h}(k+s)_{2 m-h}}{(k)_{2 m}} \\
& -\sum_{h=\nu-1}^{m-1}(-1)^{h-\nu}\left(\begin{array}{c}
h+1 \\
\nu
\end{array}\right) \frac{(k+2 m-1)(2 k+2 h-2)_{2 m-2 h-1}}{2 m(h) !(2 m-2 h-2) !(k+h-1)_{2 m-h}} \\
& \times \frac{(-s)_{h}(k+s)_{2 m-h-1}}{(k)_{2 m}}
\end{aligned}
$$




$$
\begin{aligned}
& =a(2 m, \nu, k, s)-(-1)^{m-\nu}\left(\begin{array}{c}
m \\
\nu
\end{array}\right) \frac{1}{m !(k+m-1)_{m}} \frac{(-s)_{m}(k+s)_{m}}{(k)_{2 m}} \\
& +\sum_{h=\nu-1}^{m-1}(-1)^{h-\nu}\left(\begin{array}{c}
h+1 \\
\nu
\end{array}\right) \frac{(2 k+2 h-2)_{2 m-2 h-1}}{2 m(h) !(2 m-2 h-2) !(k+h-1)_{2 m-h}} \\
& \times \frac{(-s)_{h}(k+s)_{2 m-h}}{(k)_{2 m}} \\
& -\sum_{h=\nu}^{m-1}(-1)^{h-\nu}\left(\begin{array}{l}
h \\
\nu
\end{array}\right) \frac{(2 k+2 h-3)_{2 m-2 h}}{m(h-1) !(2 m-2 h) !(k+h-1)_{2 m-h}} \\
& \times \frac{(-s)_{h}(k+s)_{2 m-h}}{(k)_{2 m}} \\
& -\sum_{h=\nu-1}^{m-1}(-1)^{h-\nu}\left(\begin{array}{c}
h+1 \\
\nu
\end{array}\right) \frac{(k+2 m-1)(2 k+2 h-2)_{2 m-2 h-1}}{2 m(h) !(2 m-2 h-2) !(k+h-1)_{2 m-h}} \\
& \times \frac{(-s)_{h}(k+s)_{2 m-h-1}}{(k)_{2 m}} \\
& =a(2 m, \nu, k, s)-(-1)^{m-\nu}\left(\begin{array}{c}
m \\
\nu
\end{array}\right) \frac{1}{m !(k+m-1)_{m}} \frac{(-s)_{m}(k+s)_{m}}{(k)_{2 m}} \\
& -\sum_{h=\nu}^{m-1}(-1)^{h-\nu}\left(\begin{array}{l}
h \\
\nu
\end{array}\right) \frac{(2 k+2 h-3)_{2 m-2 h}}{m(h-1) !(2 m-2 h) !(k+h-1)_{2 m-h}} \\
& \times \frac{(-s)_{h}(k+s)_{2 m-h}}{(k)_{2 m}} \\
& +\sum_{h=\nu-1}^{m-1}(-1)^{h+1-\nu}\left(\begin{array}{c}
h+1 \\
\nu
\end{array}\right) \frac{(2 k+2 h-1)_{2 m-2 h-2}}{m(h) !(2 m-2 h-2) !(k+h)_{2 m-h-1}} \\
& \times \frac{(-s)_{h+1}(k+s)_{2 m-h-1}}{(k)_{2 m}} \\
& =a(2 m, \nu, k, s) \text {. }
\end{aligned}
$$

\subsection{Pullback formula}

We consider $\Gamma^{n} \times \Gamma^{n}$ imbedded in $\Gamma^{2 n}$ by

$$
\left(\begin{array}{ll}
A^{(n)} & B^{(n)} \\
C^{(n)} & D^{(n)}
\end{array}\right) \times\left(\begin{array}{ll}
A^{\prime(n)} & B^{\prime(n)} \\
C^{\prime(n)} & D^{\prime(n)}
\end{array}\right) \longmapsto\left(\begin{array}{cccc}
A & 0 & B & 0 \\
0 & A^{\prime} & 0 & B^{\prime} \\
C & 0 & D & 0 \\
0 & C^{\prime} & 0 & D^{\prime}
\end{array}\right)
$$


and when convenient will identify $\Gamma^{n} \times \Gamma^{n}$ with its image in $\Gamma^{2 n}$.

In this section we decompose $\left(L^{k, l} E_{k}^{2 n}\right)(\mathfrak{Z}, s), \mathfrak{Z}=\left(\begin{array}{ll}Z^{(n)} & { }^{t} U^{(n)} \\ U^{(n)} & W^{(n)}\end{array}\right) \in \mathfrak{H}_{2 n}$ into functions for $Z$ and for $W$. The main tool is the coset decomposition by Garrett [12]:

(2.2.1). (i) The double coset $P_{2 n, 0} \backslash \Gamma^{2 n} / \Gamma^{n} \times \Gamma^{n}$ has an irredundant set of coset representatives

$$
g_{\tilde{T}}=\left(\begin{array}{cccc}
1_{n} & 0 & 0 & 0 \\
0 & 1_{n} & 0 & 0 \\
0 & \tilde{T}^{(n)} & 1_{n} & 0 \\
\tilde{T}^{(n)} & 0 & 0 & 1_{n}
\end{array}\right)
$$

where $\tilde{T}=\left(\begin{array}{cc}0 & 0 \\ 0 & T^{(r)}\end{array}\right), T \in \mathbb{T}^{(r)}(0 \leq r \leq n)$.

(ii) The left coset $P_{2 n, 0} \backslash P_{2 n, 0} g_{\tilde{T}}\left(\Gamma^{n} \times \Gamma^{n}\right)$ has an irredundant set of coset representatives $g_{\tilde{T}} \tilde{g}_{1} g_{2} \tilde{g}_{1}^{\prime} g_{2}^{\prime}$,

$$
\tilde{g}_{1} \in G_{n, r}, g_{2} \in P_{n, r} \backslash \Gamma^{n}, \tilde{g}_{1}^{\prime} \in \Gamma^{r}(T) \backslash G_{n, r}, g_{2}^{\prime} \in P_{n, r} \backslash \Gamma^{n},
$$

where

$$
G_{n, r}:=\left\{\tilde{g}=\left(\begin{array}{cc}
\tilde{A}^{(n)} & \tilde{B}^{(n)} \\
\tilde{C}^{(n)} & \tilde{D}^{(n)}
\end{array}\right)=\left(\begin{array}{cccc}
1_{n-r} & 0 & 0 & 0 \\
0 & A^{(r)} & 0 & B^{(r)} \\
0 & 0 & 1_{n-r} & 0 \\
0 & C^{(r)} & 0 & D^{(r)}
\end{array}\right) \in \Gamma^{n}\right.
$$

$$
\left.g=\left(\begin{array}{ll}
A & B \\
C & D
\end{array}\right) \in \Gamma^{r}\right\}
$$

and for $T \in \mathbb{T}^{(r)}$,

$$
\Gamma^{r}(T):=\left\{g \in \Gamma^{r} \mid\left(\begin{array}{cc}
0 & T^{-1} \\
T & 0
\end{array}\right) g\left(\begin{array}{cc}
0 & T^{-1} \\
T & 0
\end{array}\right) \in \Gamma^{r}\right\} .
$$

Then the pullback formula is given by the following:

Proposition 2. Let $k, l \in 2 \mathbb{Z}, k, l>0, s \in \mathbb{C}$ and $k+2 \operatorname{Re}(s)>2 n+1$. For $\mathfrak{Z}=\left(\begin{array}{ll}Z^{(n)} & { }^{t} U^{(n)} \\ U^{(n)} & W^{(n)}\end{array}\right) \in \mathfrak{H}_{2 n}$, we have

$$
\begin{aligned}
& \left(L^{k, l} E_{k}^{2 n}\right)(\mathfrak{Z}, s) \\
& \quad=\sum_{\nu=0}^{\frac{l}{2}}\left(-\frac{1}{4}\right)^{\nu} \frac{a(l, \nu, k, s)}{(2 \pi i)^{l}} \sum_{r=1}^{n} \sum_{T \in \mathbb{T}^{(r)}} \mathcal{P}_{\nu}\left(Z, W,\left(\begin{array}{cc}
0 & 0 \\
0 & T^{(r)}
\end{array}\right), s\right),
\end{aligned}
$$


with

$$
\begin{aligned}
\mathcal{P}_{\nu}( & \left.Z, W,\left(\begin{array}{cc}
0 & 0 \\
0 & T
\end{array}\right), s\right) \\
:= & \sum_{g_{2} \in P_{n, r} \backslash \Gamma^{n}} \sum_{g_{2}^{\prime} \in P_{n, r} \backslash \Gamma^{n}} \sum_{\tilde{g}_{1} \in G_{n, r}} \sum_{\tilde{g}_{1}^{\prime} \in \Gamma^{r}(T) \backslash G_{n, r}} \\
& \left\{\operatorname{det}\left(\operatorname{Im}\left(\tilde{g}_{1} g_{2}\langle Z\rangle\right)\right)^{s} \operatorname{det}\left(\operatorname{Im}\left(\tilde{g}_{1}^{\prime} g_{2}^{\prime}\langle W\rangle\right)\right)^{s}\right. \\
& \times\left|\operatorname{det}\left(1_{n}-\tilde{T}\left(\tilde{g}_{1}^{\prime} g_{2}^{\prime}\langle W\rangle\right) \tilde{T}\left(\tilde{g}_{1} g_{2}\langle Z\rangle\right)\right)\right|^{-2 s} \\
& \times \rho_{1}\left(\left(C_{2} Z+D_{2}\right)^{-1}\left(\tilde{C}_{1}\left(g_{2}\langle Z\rangle\right)+\tilde{D}_{1}\right)^{-1}\left(1_{n}-\tilde{T}\left(\tilde{g}_{1}^{\prime} g_{2}^{\prime}\langle W\rangle\right) \tilde{T}\left(\tilde{g}_{1} g_{2}\langle Z\rangle\right)\right)^{-1}\right) \\
& \times \rho\left(\left(C_{2}^{\prime} W+D_{2}^{\prime}\right)^{-1}\left(\tilde{C}_{1}^{\prime}\left(g_{2}^{\prime}\langle W\rangle\right)+\tilde{D}_{2}^{\prime}\right)^{-1}\right)\left(e_{1} \tilde{T}{ }^{t} e_{2}\right)^{l-2 \nu} \\
& \times\left(e_{1}\left(1_{n}-\tilde{T}\left(\tilde{g}_{1}^{\prime} g_{2}^{\prime}\langle W\rangle\right) \tilde{T}\left(\tilde{g}_{1} g_{2}\langle\bar{Z}\rangle\right)\right)\left(\operatorname{Im}\left(\tilde{g}_{1} g_{2}\langle Z\rangle\right)\right)^{-1}\right. \\
& \left.\times{ }^{t}\left(1_{n}-\tilde{T}\left(\tilde{g}_{1}^{\prime} g_{2}^{\prime}\langle W\rangle\right) \tilde{T}\left(\tilde{g}_{1} g_{2}\langle Z\rangle\right)\right)^{t} e_{1}\right)^{\nu} \\
& \times\left(e_{2}\left(1_{n}-\tilde{T}\left(\tilde{g}_{1} g_{2}\langle Z\rangle\right) \tilde{T}\left(\tilde{g}_{1}^{\prime} g_{2}^{\prime}\langle W\rangle\right)\right)^{-1}\left(1_{n}-\tilde{T}\left(\tilde{g}_{1} g_{2}\langle Z\rangle\right) \tilde{T}\left(\tilde{g}_{1}^{\prime} g_{2}^{\prime}\langle\bar{W}\rangle\right)\right)\right. \\
& \left.\left.\times\left(\operatorname{Im}\left(\tilde{g}_{1}^{\prime} g_{2}^{\prime}\langle W\rangle\right)\right)^{-1}{ }^{t} e_{2}\right)^{\nu}\right\},
\end{aligned}
$$

where $\rho_{1}=\operatorname{det}^{k} \otimes \operatorname{sym}^{l}$ (resp. $\left.\rho=\operatorname{det}^{k} \otimes \operatorname{sym}^{l}\right)$ is the representation of $G L(n, \mathbb{C})$ with the representation space $\operatorname{sym}^{l}\left(V_{1}\right)\left(\right.$ resp. $\left.\operatorname{sym}^{l}\left(V_{2}\right)\right), \tilde{g}_{1}=\left(\begin{array}{ll}\tilde{A}_{1}^{(n)} & \tilde{B}_{1}^{(n)} \\ \tilde{C}_{1}^{(n)} & \tilde{D}_{1}^{(n)}\end{array}\right)$, $\tilde{g}_{1}^{\prime}=\left(\begin{array}{ll}\tilde{A}_{1}^{\prime(n)} & \tilde{B}_{1}^{\prime(n)} \\ \tilde{C}_{1}^{\prime(n)} & \tilde{D}_{1}^{\prime(n)}\end{array}\right)$ are of the form $(2.12), g_{2}=\left(\begin{array}{ll}A_{2}{ }^{(n)} & B_{2}{ }^{(n)} \\ C_{2}{ }^{(n)} & D_{2}{ }^{(n)}\end{array}\right)$, $g_{2}^{\prime}=\left(\begin{array}{ll}A_{2}^{\prime(n)} & B_{2}^{\prime(n)} \\ C_{2}^{\prime(n)} & D_{2}^{\prime(n)}\end{array}\right), \quad \tilde{T}^{(n)}=\left(\begin{array}{cc}0 & 0 \\ 0 & T^{(r)}\end{array}\right)$.

Proof. By Proposition 1, we have only to prove

$$
\begin{gathered}
\sum_{M} \operatorname{det}\left(\mathfrak{C}_{0}+\mathfrak{D}\right)^{-k} \operatorname{det}\left(\operatorname{Im}\left(M\left\langle\mathfrak{Z}_{0}\right\rangle\right)\right)^{s}{Q_{0}}^{l-2 \nu}\left(P_{0}-P_{0}^{\prime}\right)^{\nu}\left(R_{0}-R_{0}^{\prime}\right)^{\nu} \\
=\left(-\frac{1}{4}\right)^{\nu} \sum_{r=1}^{n} \sum_{T \in \mathbb{T}^{(r)}} \mathcal{P}_{\nu}\left(Z, W,\left(\begin{array}{cc}
0 & 0 \\
0 & T
\end{array}\right), s\right) \quad\left(0 \leq \nu \leq \frac{l}{2}\right)
\end{gathered}
$$

where the notations are the same as those in Proposition 1. 
We put $g=\left(\begin{array}{ll}A^{(n)} & B^{(n)} \\ C^{(n)} & D^{(n)}\end{array}\right)=\tilde{g}_{1} g_{2}, \quad g^{\prime}=\left(\begin{array}{ll}A^{(n)} & B^{\prime(n)} \\ C^{(n)} & D^{\prime(n)}\end{array}\right)=\tilde{g}_{1}^{\prime} g_{2}^{\prime}$. Then a coset representative $M=\left(\begin{array}{ll}\mathfrak{A}^{(2 n)} & \mathfrak{B}^{(2 n)} \\ \mathfrak{C}^{(2 n)} & \mathfrak{D}^{(2 n)}\end{array}\right)$ of $P_{2 n, 0} \backslash \Gamma^{2 n}$ can be written in the following form:

$$
M=g_{\tilde{T}} g g^{\prime}=\left(\begin{array}{cccc}
A & 0 & B & 0 \\
0 & A^{\prime} & 0 & B^{\prime} \\
C & \tilde{T} A^{\prime} & D & \tilde{T} B^{\prime} \\
\tilde{T} A & C^{\prime} & \tilde{T} B & D^{\prime}
\end{array}\right) .
$$

By $\left(\operatorname{Im}\left(\mathfrak{Z}_{0}\right)\right)^{-1}=\left(\mathfrak{C} \mathfrak{Z}_{0}+\mathfrak{D}\right)^{-1}\left(\operatorname{Im}\left(M\left\langle\mathfrak{Z}_{0}\right\rangle\right)\right)^{-1}{ }^{t}\left(\mathfrak{C} \mathfrak{Z}_{0}+\mathfrak{D}\right)^{-1}+2 i\left(\mathfrak{C} \mathfrak{Z}_{0}+\mathfrak{D}\right)^{-1} \mathfrak{C}$, we have

$$
\begin{aligned}
& P_{0}-P_{0}^{\prime}=\left(-\frac{1}{2 i}\right)\left(\left(\mathfrak{C} \mathfrak{Z}_{0}+\mathfrak{D}\right)^{-1}\left(\mathfrak{C} \overline{\mathfrak{Z}}_{0}+\mathfrak{D}\right)\left(\operatorname{Im}\left(\mathfrak{Z}_{0}\right)\right)^{-1}\right)\left[\left(\begin{array}{c}
{ }^{t} e_{1} \\
0
\end{array}\right)\right] \\
& R_{0}-R_{0}^{\prime}=\left(-\frac{1}{2 i}\right)\left(\left(\mathfrak{C} \mathfrak{Z}_{0}+\mathfrak{D}\right)^{-1}\left(\mathfrak{C} \overline{\mathfrak{Z}}_{0}+\mathfrak{D}\right)\left(\operatorname{Im}\left(\mathfrak{Z}_{0}\right)\right)^{-1}\right)\left[\left(\begin{array}{c}
0 \\
{ }^{t} e_{2}
\end{array}\right)\right]
\end{aligned}
$$

On the other hand, we have

$$
\mathfrak{C} \mathfrak{Z}_{0}+\mathfrak{D}=\left(\begin{array}{cc}
C Z+D & \tilde{T}\left(A^{\prime} W+B^{\prime}\right) \\
\tilde{T}(A Z+B) & C^{\prime} W+D^{\prime}
\end{array}\right)
$$

and

$$
\begin{aligned}
& \left(\mathfrak{C} \mathfrak{Z}_{0}+\mathfrak{D}\right)^{-1} \\
& =\left(\begin{array}{cc}
(C Z+D)^{-1} & -(C Z+D)^{-1} \\
\times\left(1_{n}-\tilde{T} g^{\prime}\langle W\rangle \tilde{T} g\langle Z\rangle\right)^{-1} & \times\left(1_{n}-\tilde{T} g^{\prime}\langle W\rangle \tilde{T} g\langle Z\rangle\right)^{-1} \tilde{T} g^{\prime}\langle W\rangle \\
-\left(C^{\prime} W+D^{\prime}\right)^{-1} & \\
\times\left(1_{n}-\tilde{T} g\langle Z\rangle \tilde{T} g^{\prime}\langle W\rangle\right)^{-1} \tilde{T} g\langle Z\rangle & \times\left(1_{n}-W+D^{\prime}\right)^{-1} \\
&
\end{array}\right) .
\end{aligned}
$$

If we put $\left(\mathfrak{C} \mathfrak{Z}_{0}+\mathfrak{D}\right)^{-1}\left(\mathfrak{C} \overline{\mathfrak{Z}}_{0}+\mathfrak{D}\right)\left(\operatorname{Im}\left(\mathfrak{Z}_{0}\right)\right)^{-1}=\left(\begin{array}{cc}\mathcal{A}^{(n)} & * \\ * & \mathcal{D}^{(n)}\end{array}\right)$ and $\left(\mathfrak{C} \mathfrak{Z}_{0}+\mathfrak{D}\right)^{-1} \mathfrak{C}=$ $\left(\begin{array}{cc}* & \mathcal{B}^{(n)} \\ * & *\end{array}\right)$, we get

$$
\begin{aligned}
& \mathcal{A}=(C Z+D)^{-1}\left(1_{n}-\tilde{T} g^{\prime}\langle W\rangle \tilde{T} g\langle Z\rangle\right)^{-1}\left(1_{n}-\tilde{T} g^{\prime}\langle W\rangle \tilde{T} g\langle\bar{Z}\rangle\right) \\
& \times(C \bar{Z}+D)(\operatorname{Im}(Z))^{-1}, \\
& \mathcal{D}=\left(C^{\prime} W+D^{\prime}\right)^{-1}\left(1_{n}-\tilde{T} g\langle Z\rangle \tilde{T} g^{\prime}\langle W\rangle\right)^{-1}\left(1_{n}-\tilde{T} g\langle Z\rangle \tilde{T} g^{\prime}\langle\bar{W}\rangle\right) \\
& \quad \times\left(C^{\prime} \bar{W}+D^{\prime}\right)(\operatorname{Im}(W))^{-1}, \\
& \mathcal{B}=(C Z+D)^{-1}\left(1_{n}-\tilde{T} g^{\prime}\langle W\rangle \tilde{T} g\langle Z\rangle\right)^{-1} \tilde{T}^{t}\left(C^{\prime} W+D^{\prime}\right)^{-1}
\end{aligned}
$$


Thus, if we note

$$
\begin{gathered}
g\langle Z\rangle=\tilde{g}_{1} g_{2}\langle Z\rangle, \quad C Z+D=\left(\tilde{C}_{1}\left(g_{2}\langle Z\rangle\right)+\tilde{D}_{1}\right)\left(C_{2} Z+D_{2}\right), \\
g^{\prime}\langle W\rangle=\tilde{g}_{1}^{\prime} g_{2}^{\prime}\langle W\rangle, \quad C^{\prime} W+D^{\prime}=\left(\tilde{C}_{1}^{\prime}\left(g_{2}^{\prime}\langle W\rangle\right)+\tilde{D}_{1}^{\prime}\right)\left(C_{2}^{\prime} W+D_{2}^{\prime}\right),
\end{gathered}
$$

we get

$$
\begin{aligned}
& Q_{0}=e_{1}\left(C_{2} Z+D_{2}\right)^{-1}\left(\tilde{C}_{1}\left(g_{2}\langle Z\rangle\right)+\tilde{D}_{1}\right)^{-1}\left(1_{n}-\tilde{T}\left(\tilde{g}_{1}^{\prime} g_{2}^{\prime}\langle W\rangle\right) \tilde{T}\left(\tilde{g}_{1} g_{2}\langle Z\rangle\right)\right)^{-1} \\
& \times \tilde{T}^{t}\left(\tilde{C}_{1}^{\prime}\left(g_{2}^{\prime}\langle W\rangle\right)+\tilde{D}_{1}^{\prime}\right)^{-1}{ }^{t}\left(C_{2}^{\prime} W+D_{2}^{\prime}\right)^{-1}{ }^{t} e_{2} \\
& P_{0}-P_{0}^{\prime}=\left(-\frac{1}{2 i}\right) e_{1}\left(C_{2} Z+D_{2}\right)^{-1}\left(\tilde{C}_{1}\left(g_{2}\langle Z\rangle\right)+\tilde{D}_{1}\right)^{-1} \\
& \times\left(1_{n}-\tilde{T}\left(\tilde{g}_{1}^{\prime} g_{2}^{\prime}\langle W\rangle\right) \tilde{T}\left(\tilde{g}_{1} g_{2}\langle Z\rangle\right)\right)^{-1} \\
& \times\left(1_{n}-\tilde{T}\left(\tilde{g}_{1}^{\prime} g_{2}^{\prime}\langle W\rangle\right) \tilde{T}\left(\tilde{g}_{1} g_{2}\langle\bar{Z}\rangle\right)\right)\left(\tilde{C}_{1}\left(g_{2}\langle\bar{Z}\rangle\right)+\tilde{D}_{1}\right) \\
& \times\left(C_{2} \bar{Z}+D_{2}\right)(\operatorname{Im}(Z))^{-1}{ }^{t} e_{1}, \\
& R_{0}-R_{0}^{\prime}=\left(-\frac{1}{2 i}\right) e_{2}\left(C_{2}^{\prime} W+D_{2}^{\prime}\right)^{-1}\left(\tilde{C}_{1}^{\prime}\left(g_{2}^{\prime}\langle W\rangle\right)+\tilde{D}_{1}^{\prime}\right)^{-1} \\
& \times\left(1_{n}-\tilde{T}\left(\tilde{g}_{1} g_{2}\langle Z\rangle\right) \tilde{T}\left(\tilde{g}_{1}^{\prime} g_{2}^{\prime}\langle W\rangle\right)\right)^{-1} \\
& \times\left(1_{n}-\tilde{T}\left(\tilde{g}_{1} g_{2}\langle Z\rangle\right) \tilde{T}\left(\tilde{g}_{1}^{\prime} g_{2}^{\prime}\langle\bar{W}\rangle\right)\right)\left(\tilde{C}_{1}^{\prime}\left(g_{2}^{\prime}\langle\bar{W}\rangle\right)+\tilde{D}_{1}^{\prime}\right) \\
& \times\left(C_{2}^{\prime} \bar{W}+D_{2}^{\prime}\right)(\operatorname{Im}(W))^{-1} e_{2}
\end{aligned}
$$

and

$$
\begin{aligned}
& \operatorname{det}\left(\mathfrak{C} \mathfrak{Z}_{0}+\mathfrak{D}\right)^{-k} \operatorname{det}\left(\operatorname{Im}\left(M\left\langle\mathfrak{Z}_{0}\right\rangle\right)\right)^{s} \\
& =\operatorname{det}\left(\mathfrak{C} \mathfrak{Z}_{0}+\mathfrak{D}\right)^{-k}\left|\operatorname{det}\left(\mathfrak{C} \mathfrak{Z}_{0}+\mathfrak{D}\right)\right|^{-2 s} \operatorname{det}\left(\operatorname{Im}\left(\mathfrak{Z}_{0}\right)\right)^{s} \\
& =\operatorname{det}\left(\tilde{C}_{1}\left(g_{2}\langle Z\rangle\right)+\tilde{D}_{1}\right)^{-k} \operatorname{det}\left(C_{2} Z+D_{2}\right)^{-k} \operatorname{det}\left(\tilde{C}_{1}^{\prime}\left(g_{2}^{\prime}\langle W\rangle\right)+\tilde{D}_{1}^{\prime}\right)^{-k} \\
& \quad \times \operatorname{det}\left(C_{2}^{\prime} W+D_{2}^{\prime}\right)^{-k} \operatorname{det}\left(1_{n}-\tilde{T}\left(\tilde{g}_{1}^{\prime} g_{2}^{\prime}\langle W\rangle\right) \tilde{T}\left(\tilde{g}_{1} g_{2}\langle Z\rangle\right)\right)^{-k} \\
& \quad \times\left|\operatorname{det}\left(1_{n}-\tilde{T}\left(\tilde{G}_{1}^{\prime} g_{2}^{\prime}\langle W\rangle\right) \tilde{T}\left(\tilde{g}_{1} g_{2}\langle Z\rangle\right)\right)\right|^{-2 s} \operatorname{det}\left(\operatorname{Im}\left(\tilde{g}_{1} g_{2}\langle Z\rangle\right)\right)^{s} \operatorname{det}\left(\operatorname{Im}\left(\tilde{g}_{1}^{\prime} g_{2}^{\prime}\langle W\rangle\right)\right)^{s} .
\end{aligned}
$$

Combining these formulae and using the representations $\rho_{1}, \rho$, we obtain Proposition 2. 


\section{$\S 3 . \quad$ Analytic properties of standard $L$-functions}

\subsection{Integral representation}

We first prove the following:

Theorem 1. Let $k, l \in 2 \mathbb{Z}, k, l>0, s \in \mathbb{C}$ and $k+2 \operatorname{Re}(s)>2 n+1$. For an eigenform $f \in S_{k, l}^{n}\left(\operatorname{sym}^{l}\left(V_{2}\right)\right)$ and each $T \in \mathbb{T}^{(r)}(1 \leq r \leq n)$, the Petersson inner product of $f$ and $\mathcal{P}_{\nu}\left(Z, W,\left(\begin{array}{ll}0 & 0 \\ 0 & T\end{array}\right), s\right)\left(0 \leq \nu \leq \frac{l}{2}\right)$ is convergent and then,

(i) for $T \in \mathbb{T}^{(r)} \quad(1 \leq r<n)$,

$$
\left(f, \mathcal{P}_{\nu}\left(-\bar{Z}, *,\left(\begin{array}{cc}
0 & 0 \\
0 & T
\end{array}\right), \bar{s}\right)\right)=0
$$

(ii) for $T \in \mathbb{T}^{(n)}$,

$$
\begin{aligned}
(f, & \left.\left(-\frac{1}{4}\right)^{\nu} \mathcal{P}_{\nu}(-\bar{Z}, *, T, \bar{s})\right) \\
= & \lambda(f, T) \operatorname{det}(T)^{-k-2 s} 2^{n(n+1-k-2 s)-l+1} i^{n k+l} \pi^{\frac{n(n+1)}{2}}\left(\iota^{-1}(f)\right)(Z) \\
& \times \frac{(-1)^{\nu} \nu !}{(k+s+l-\nu-1)_{\nu+1}} \sum_{j=1}^{n-1} \frac{\Gamma(2 k+2 s-2 n-1+2 j)(2 k+2 s-n-2+j)_{l}}{(k+s-n-1+j) \Gamma(2 k+2 s+l-n-1+j)} .
\end{aligned}
$$

Proof. It follows from (1.4.2) that $\left(f,\left(L^{k, l} E_{k}^{2 n}\right)\left(\left(\begin{array}{cc}-\bar{Z}^{(n)} & 0 \\ 0 & *\end{array}\right), \bar{s}\right)\right)$ converges absolutely and locally uniformly for $k+2 \operatorname{Re}(s)>2 n+1$. Then, by Proposition 2, $\left(f, \mathcal{P}_{\nu}\left(-\bar{Z}, *,\left(\begin{array}{cc}0 & 0 \\ 0 & T\end{array}\right), \bar{s}\right)\right)$ is convergent.

The assertion (i) is proved in the same way as that by Klingen [17, Satz 2]:

We can write

$$
\begin{aligned}
\mathcal{P}_{\nu}\left(Z, W,\left(\begin{array}{ll}
0 & 0 \\
0 & T
\end{array}\right), s\right) \\
=\sum_{g_{2} \in P_{n, r} \backslash \Gamma^{n}} \sum_{g_{2}^{\prime} \in P_{n, r} \backslash \Gamma^{n}} \sum_{\tilde{g}_{1} \in G_{n, r}} \sum_{\tilde{g}_{1}^{\prime} \in \Gamma^{r}(T) \backslash G_{n, r}}\left\{\operatorname{det}(\operatorname{Im}(Z))^{s} \operatorname{det}(\operatorname{Im}(W))^{s}\right. \\
\quad \times\left|\operatorname{det}\left(1_{n}-\tilde{T} W \tilde{T} Z\right)\right|^{-2 s} \rho_{1}\left(\left(1_{n}-\tilde{T} W \tilde{T} Z\right)^{-1}\right)\left(e_{1} \tilde{T}^{t} e_{2}\right)^{l-2 \nu} \\
\quad \times\left(e_{1}\left(1_{n}-\tilde{T} W \tilde{T} \bar{Z}\right) \operatorname{Im}(Z)^{-1} t^{t}\left(1_{n}-\tilde{T} W \tilde{T} Z\right)^{t} e_{1}\right)^{\nu}
\end{aligned}
$$




$$
\begin{gathered}
\left.\times\left(e_{2}\left(1_{n}-\tilde{T} Z \tilde{T} W\right)^{-1}\left(1_{n}-\tilde{T} Z \tilde{T} \bar{W}\right) \operatorname{Im}(W)^{-1}{ }^{t} e_{2}\right)^{\nu}\right\} \\
\left|\left(\tilde{g}_{1}^{\prime}\right)_{W}\right|\left(\tilde{g}_{1}\right)_{Z}\left|\left(g_{2}^{\prime}\right)_{W}\right|\left(g_{2}\right)_{Z},
\end{gathered}
$$

where ()$_{Z}$ (resp. ()$\left._{W}\right)$ denotes the action on $Z$ (resp. $W$ ). Then we put

$$
\begin{aligned}
G_{\nu}\left(Z, W,\left(\begin{array}{cc}
0 & 0 \\
0 & T
\end{array}\right), s\right) \\
:=\operatorname{det}(\operatorname{Im}(Z))^{s} \operatorname{det}(\operatorname{Im}(W))^{s}\left|\operatorname{det}\left(1_{n}-\tilde{T} W \tilde{T} Z\right)\right|^{-2 s} \\
\quad \times \rho_{1}\left(\left(1_{n}-\tilde{T} W \tilde{T} Z\right)^{-1}\right)\left(e_{1} \tilde{T}^{t} e_{2}\right)^{l-2 \nu} \\
\quad \times\left(e_{1}\left(1_{n}-\tilde{T} W \tilde{T} \bar{Z}\right) \operatorname{Im}(Z)^{-1}{ }^{t}\left(1_{n}-\tilde{T} W \tilde{T} Z\right)^{t} e_{1}\right)^{\nu} \\
\times\left(e_{2}\left(1_{n}-\tilde{T} Z \tilde{T} W\right)^{-1}\left(1_{n}-\tilde{T} Z \tilde{T} \bar{W}\right) \operatorname{Im}(W)^{-1}{ }^{t} e_{2}\right)^{\nu}
\end{aligned}
$$

Now, we put

$$
\begin{gathered}
W=X+i Y=\left(\begin{array}{cc}
W_{1}^{(n-r)} & { }^{t} W_{2}^{(n-r, r)} \\
W_{2}^{(r, n-r)} & W_{*}^{(r)}
\end{array}\right), \\
X=\left(\begin{array}{cc}
X_{1}^{(n-r)} & { }^{t} X_{2}^{(n-r, r)} \\
X_{2}^{(r, n-r)} & X_{*}^{(r)}
\end{array}\right), \quad Y=\left(\begin{array}{cc}
Y_{1}^{(n-r)} & 0 \\
0 & Y_{*}^{(r)}
\end{array}\right)\left[\left(\begin{array}{cc}
1_{n-r} & 0 \\
Y_{2}^{(r, n-r)} & 1_{r}
\end{array}\right)\right] .
\end{gathered}
$$

Let $F_{n}$ be a fundamental domain of $\Gamma^{n} \backslash \mathfrak{H}_{n}$ and $F_{n, r}$ be a fundamental domain of $P_{n, r} \backslash \mathfrak{H}_{n}$, that is,

$$
F_{n, r}:=\left\{W \in \mathfrak{H}_{n} \mid W_{*} \in F_{r}, Y_{1} \in M_{n-r}, X \text { and } Y_{2} \text { are reduced } \bmod 1\right\}
$$

where $M_{n-r}$ is the Minkowski reduction domain of the positive definite quadratic forms of degree $n-r$. Then we obtain

$$
\begin{gathered}
\left(f, \mathcal{P}_{\nu}\left(-\bar{Z}, *,\left(\begin{array}{cc}
0 & 0 \\
0 & T
\end{array}\right), \bar{s}\right)\right) \\
=\int_{F_{n}}\left\langle\rho(\sqrt{\operatorname{Im}(W)}) f(W), \rho(\sqrt{\operatorname{Im}(W)}) \mathcal{P}_{\nu}\left(-\bar{Z}, W,\left(\begin{array}{cc}
0 & 0 \\
0 & T
\end{array}\right), \bar{s}\right)\right\rangle \\
\times \operatorname{det}(\operatorname{Im}(W))^{-n-1} d W
\end{gathered}
$$




$$
\begin{aligned}
& =2^{-1} \int_{F_{n, r}}\langle\rho(\sqrt{\operatorname{Im}(W)}) f(W), \\
& \sum_{g_{2}^{\prime}} \sum_{\tilde{g}_{1}} \sum_{\tilde{g}_{1}^{\prime}} \rho(\sqrt{\operatorname{Im}(W)}) G_{\nu}\left(-\bar{Z}, W,\left(\begin{array}{cc}
0 & 0 \\
0 & T
\end{array}\right), \bar{s}\right)\left|\left(\tilde{g}_{1}^{\prime}\right)_{W}\right|\left(\tilde{g}_{1}\right)_{Z}\left|\left(g_{2}^{\prime}\right)_{W}\right\rangle \\
& \times \operatorname{det}(\operatorname{Im}(W))^{-n-1} d W .
\end{aligned}
$$

Here we recall that $\tilde{g}_{1}^{\prime}$ is of the form

$$
\left(\begin{array}{ll}
\tilde{A}_{1}^{\prime(n)} & \tilde{B}_{1}^{\prime(n)} \\
\tilde{C}_{1}^{\prime(n)} & \tilde{D}_{1}^{\prime(n)}
\end{array}\right)=\left(\begin{array}{cccc}
1_{n-r} & 0 & 0 & 0 \\
0 & A_{1}^{\prime(r)} & 0 & B_{1}^{\prime(r)} \\
0 & 0 & 1_{n-r} & 0 \\
0 & C_{1}^{\prime(r)} & 0 & D_{1}^{\prime(r)}
\end{array}\right) \quad \text { with } \quad g_{1}^{\prime}=\left(\begin{array}{cc}
A_{1}^{\prime} & B_{1}^{\prime} \\
C_{1}^{\prime} & D_{1}^{\prime}
\end{array}\right)
$$

Since

$$
\tilde{g}_{1}^{\prime}\langle W\rangle=\left(\begin{array}{cc}
W_{1}+{ }^{t} W_{2}\left(C_{1}^{\prime} W_{*}+D_{1}^{\prime}\right)^{-1} C_{1}^{\prime} W_{2} & { }^{t} W_{2}\left(C_{1}^{\prime} W_{*}+D_{1}^{\prime}\right)^{-1} \\
A_{1}^{\prime} W_{2}+\left(g_{1}^{\prime}\left\langle W_{*}\right\rangle\right) C_{1}^{\prime} W_{2} & g_{1}^{\prime}\left\langle W_{*}\right\rangle
\end{array}\right),
$$

each of $\tilde{T}\left(\tilde{g}_{1}^{\prime}\langle W\rangle\right) \tilde{T}, \tilde{T}\left(\tilde{g}_{1}^{\prime}\langle W\rangle\right), \tilde{C}_{1}^{\prime} W+\tilde{D}_{1}^{\prime}$ as a function on $W$ does not depend on $W_{1}$. Therefore,

$$
\sum_{g_{2}^{\prime}} \sum_{\tilde{g}_{1}} \sum_{\tilde{g}_{1}^{\prime}} \rho(\sqrt{\operatorname{Im}(W)}) G_{\nu}\left(-\bar{Z}, W,\left(\begin{array}{cc}
0 & 0 \\
0 & T
\end{array}\right), \bar{s}\right)\left|\left(\tilde{g}_{1}^{\prime}\right)_{W}\right|\left(\tilde{g}_{1}\right)_{Z} \mid\left(g_{2}^{\prime}\right)_{W}
$$

is a constant function on $X_{1}$. On the other hand, since $f$ is a cusp form, its Fourier expansion has no constant terms in $X_{1}$. If we consider an integral of the integrand above on a unit cube in $X_{1}$, we find that it vanishes. Thus the the assertion (i) is proved. get

$$
\text { Proof of (ii). By } \Gamma^{n}\left(\begin{array}{cc}
T & 0 \\
0 & T^{-1}
\end{array}\right) \Gamma^{n}=\bigcup_{g^{\prime} \in \Gamma^{n}(T) \backslash \Gamma^{n}} \Gamma^{n}\left(\begin{array}{cc}
T & 0 \\
0 & T^{-1}
\end{array}\right) g^{\prime} \text {, we }
$$

$$
\mathcal{P}_{\nu}(Z, W, T, s)=\left(\mathcal{P}_{\nu}\left(Z, W, 1_{n}, s\right) \mid\left(\Gamma^{n}\left(\begin{array}{cc}
T & 0 \\
0 & T^{-1}
\end{array}\right) \Gamma^{n}\right)_{W}\right) \operatorname{det}(T)^{-k-2 s},
$$

where

$$
\begin{aligned}
& \mathcal{P}_{\nu}\left(Z, W, 1_{n}, s\right) \\
& \quad:=\sum_{g \in \Gamma^{n}}\left\{\operatorname{det}(\operatorname{Im}(Z))^{s} \operatorname{det}(\operatorname{Im}(W))^{s}|\operatorname{det}(W+Z)|^{-2 s} \rho_{1}\left((W+Z)^{-1}\right)\right.
\end{aligned}
$$




$$
\begin{aligned}
& \times\left(e_{1}{ }^{t} e_{2}\right)^{l-2 \nu}\left(e_{1}(W+\bar{Z}) \operatorname{Im}(Z)^{-1}(W+Z)^{t} e_{1}\right)^{\nu} \\
& \left.\times\left(e_{2}(W+Z)^{-1}(\bar{W}+Z) \operatorname{Im}(W)^{-1}{ }^{t} e_{2}\right)^{\nu}\right\} \mid(g)_{Z}
\end{aligned}
$$

Since the Hecke operator is an Hermitian operator and $f$ is an eigenform, we have

$$
\begin{aligned}
&(f,\left.\left(-\frac{1}{4}\right)^{\nu} \mathcal{P}_{\nu}(-\bar{Z}, *, T, \bar{s})\right) \\
&=\lambda(f, T) \operatorname{det}(T)^{-k-2 s}(-1)^{\nu} 2^{-2 \nu}\left(f, \mathcal{P}_{\nu}\left(-\bar{Z}, *, 1_{n}, \bar{s}\right)\right) \\
&=\lambda(f, T) \operatorname{det}(T)^{-k-2 s}(-1)^{\nu} 2^{-2 \nu+1} \\
& \times \int_{\mathfrak{H}_{n}}\left\langle\rho(\sqrt{\operatorname{Im}(W)}) f(W), \rho\left(\sqrt{\operatorname{Im}(W)}(W-\bar{Z})^{-1}\right)\left(e_{1}{ }^{t} e_{2}\right)^{l-2 \nu}\right. \\
& \times\left(e_{1}(W-\bar{Z})^{-1}(W-Z) \operatorname{Im}(Z)^{-1}{ }^{t} e_{1}\right)^{\nu} \\
&\left.\times\left(e_{2}(\bar{W}-\bar{Z}) \operatorname{Im}(W)^{-1}(W-\bar{Z})^{t} e_{2}\right)^{\nu}\right\rangle \\
& \times|\operatorname{det}(W-\bar{Z})|^{-2 s} \operatorname{det}(\operatorname{Im}(Z))^{s} \operatorname{det}(\operatorname{Im}(W))^{s-n-1} d W
\end{aligned}
$$

We compute the integral (3.1) according to Klingen $[16, \S 1]$ :

For $Z \in \mathfrak{H}_{n}$, there exists $F \in G L(n, \mathbb{R})$ such that $\operatorname{Im}(Z)\left[{ }^{t} F\right]=1_{n}$. Then, let $\mathcal{L}_{Z}$ be a biholomorphic transformation from $\mathfrak{H}_{n}$ onto

$$
S^{n}:=\left\{S \in \mathbb{C}^{(n)} \mid S={ }^{t} S, 1_{n}-\bar{S} S>0\right\}
$$

such that for $W \in \mathfrak{H}_{n}$,

$$
\mathcal{L}_{Z}(W)=S:=F(W-Z)(W-\bar{Z})^{-1} F^{-1} .
$$

We note

$$
\begin{gathered}
\operatorname{Im}(W)=2^{-2}(\bar{W}-Z)^{t} F\left(1_{n}-\bar{S} S\right) F(W-\bar{Z}), \\
d W=2^{-n(n+1)} \operatorname{det}(\operatorname{Im}(Z))^{-(n+1)}|\operatorname{det}(W-\bar{Z})|^{2(n+1)} d S,
\end{gathered}
$$

where $d S=d X_{S} d Y_{S}\left(S=X_{S}+i Y_{S}\right)$ is of the form (1.2). We also put

$$
\widehat{f}(S):=\rho(W-\bar{Z}) f(W)
$$

Then, the integral (3.1) is: 


$$
\begin{aligned}
& \lambda(f, T) \operatorname{det}(T)^{-k-2 s}(-1)^{\nu} 2^{n(n+1-2 s)-2(n k+l)+1} \\
& \times \int_{S^{n}}\left\langle\widehat{f}(S), \rho\left({ }^{t} F\left(1_{n}-\bar{S} S\right) F\right)\left(e_{1}{ }^{t} e_{2}\right)^{l-2 \nu}\left(e_{1}{ }^{t} F S F^{t} e_{1}\right)^{\nu}\right. \\
& \left.\quad \times\left(e_{2} F^{-1} \bar{S}\left(1_{n}-S \bar{S}\right)^{-1}{ }^{t} F^{-1}{ }^{t} e_{2}\right)^{\nu}\right\rangle \operatorname{det}\left(1_{n}-\bar{S} S\right)^{s-n-1} d S \\
& =\lambda(f, T) \operatorname{det}(T)^{-k-2 s}(-1)^{\nu} 2^{n(n+1-2 s)-2(n k+l)+1} \\
& \times \int_{S^{n}}\left\langle\rho(Z-\bar{Z}) f(Z), \rho\left({ }^{t} F\left(1_{n}-\bar{S} S\right) F\right)\left(e_{1}{ }^{t} e_{2}\right)^{l-2 \nu}\left(e_{1}{ }^{t} F S F^{t} e_{1}\right)^{\nu}\right. \\
& \left.\quad \times\left(e_{2} F^{-1} \bar{S}\left(1_{n}-S \bar{S}\right)^{-1}{ }^{t} F^{-1}{ }^{t} e_{2}\right)^{\nu}\right\rangle \operatorname{det}\left(1_{n}-\bar{S} S\right)^{s-n-1} d S
\end{aligned}
$$

by $\quad \frac{\partial}{\partial S} \operatorname{det}\left(1_{n}-S \bar{S}\right)=-\operatorname{det}\left(1_{n}-\bar{S} S\right)\left(\bar{S}\left(1_{n}-S \bar{S}\right)^{-1}\right) \quad$ with $\quad \frac{\partial}{\partial S}=$ $\left(\frac{1+\delta_{j h}}{2} \frac{\partial}{\partial s_{j h}}\right)_{1 \leq j, h \leq n}$, where $\frac{\partial}{\partial s_{j h}}$ is of the form (2.1),

$$
\begin{aligned}
& =\lambda(f, T) \operatorname{det}(T)^{-k-2 s} 2^{n(n+1-k-2 s)-l+1} i^{n k+l} \\
& \quad \times \int_{S^{n}}\left\langle\rho\left(1_{n}-\bar{S} S\right) \rho\left({ }^{t} F^{-1}\right) f(Z), \rho_{1}\left({ }^{t} F\right)\left(e_{1}{ }^{t} e_{2}\right)^{l-2 \nu}\left(e_{1} S^{t} e_{1}\right)^{\nu}\right. \\
& \left.\quad \times\left(e_{2}\left(\frac{\partial}{\partial S} \operatorname{det}\left(1_{n}-S \bar{S}\right)\right)^{t} e_{2}\right)^{\nu}\right\rangle \operatorname{det}\left(1_{n}-\bar{S} S\right)^{s-n-1-\nu} d S
\end{aligned}
$$

Moreover, there exists a linear map $\psi=\psi(n, l, \nu, k+s-n-1) \in G L\left(\operatorname{sym}^{l}\left(V_{1}\right)\right)$ such that

$$
\begin{aligned}
& \rho_{1}\left({ }^{t} \bar{F}\right) \psi \rho_{1}\left({ }^{t} F^{-1}\right)\left(\iota^{-1}(f)\right)(Z) \\
& =\int_{S^{n}}\left\langle\rho\left(1_{n}-\bar{S} S\right) \rho\left({ }^{t} F^{-1}\right) f(Z), \rho_{1}\left({ }^{t} F\right)\left(e_{1}{ }^{t} e_{2}\right)^{l-2 \nu}\left(e_{1} S^{t} e_{1}\right)^{\nu}\right. \\
& \left.\quad \times\left(e_{2}\left(\frac{\partial}{\partial S} \operatorname{det}\left(1_{n}-S \bar{S}\right)\right){ }^{t} e_{2}\right)^{\nu}\right\rangle \operatorname{det}\left(1_{n}-\bar{S} S\right)^{s-n-1-\nu} d S
\end{aligned}
$$

For any unitary matrix $U \in U(n, \mathbb{C})$, we have

$$
\begin{gathered}
\rho_{1}\left({ }^{t} \bar{F}\right) \rho_{1}\left(U^{-1}\right) \psi \rho_{1}(U) \rho_{1}\left({ }^{t} F^{-1}\right)\left(\iota^{-1}(f)\right)(Z) \\
=\rho_{1}\left({ }^{t}(\overline{U F})\right) \psi\left(\iota^{-1}\left(\rho\left(U^{t} F^{-1}\right) f\right)\right)(Z)
\end{gathered}
$$




$$
\begin{gathered}
=\int_{S^{n}}\left\langle\rho\left(1_{n}-\bar{S} S\right) \rho\left(U^{t} F^{-1}\right) f(Z), \rho_{1}\left({ }^{t}(U F)\right)\left(e_{1}{ }^{t} e_{2}\right)^{l-2 \nu}\left(e_{1} S^{t} e_{1}\right)^{\nu}\right. \\
\left.\times\left(e_{2}\left(\frac{\partial}{\partial S} \operatorname{det}\left(1_{n}-S \bar{S}\right)\right)^{t} e_{2}\right)^{\nu}\right\rangle \operatorname{det}\left(1_{n}-\bar{S} S\right)^{s-n-1-\nu} d S
\end{gathered}
$$

which equals, with the change of the variable $S$ to ${ }^{t} U S U=S^{\prime}$,

$$
\begin{aligned}
& =\int_{S^{n}}\left\langle\rho(U) \rho\left(1_{n}-\overline{S^{\prime}} S^{\prime}\right) \rho\left({ }^{t} F^{-1}\right) f(Z), \rho(U) \rho_{1}\left({ }^{t} F\right)\left(e_{1}{ }^{t} e_{2}\right)^{l-2 \nu}\left(e_{1} S^{\prime}{ }^{t} e_{1}\right)^{\nu}\right. \\
& \left.\quad \times\left(e_{2}\left(\frac{\partial}{\partial S^{\prime}} \operatorname{det}\left(1_{n}-S^{\prime} \overline{S^{\prime}}\right)\right)^{t} e_{2}\right)^{\nu}\right\rangle \operatorname{det}\left(1_{n}-\overline{S^{\prime}} S^{\prime}\right)^{s-n-1-\nu} d S^{\prime} \\
& =\rho_{1}\left({ }^{t} \bar{F}\right) \psi \rho_{1}\left({ }^{t} F^{-1}\right)\left(\iota^{-1}(f)\right)(Z),
\end{aligned}
$$

that is, $\rho_{1}\left(U^{-1}\right) \psi \rho_{1}(U)=\psi$. Since $\rho_{1}=\operatorname{det}^{k} \otimes \operatorname{sym}^{l}$ is an irreducible representation of $U(n, \mathbb{C}), \psi$ is a homothety by Schur's lemma.

Thus we get

$$
\begin{aligned}
& \left(f,\left(-\frac{1}{4}\right)^{\nu} \mathcal{P}_{\nu}(-\bar{Z}, *, T, \bar{s})\right) \\
& \quad=\lambda(f, T) \operatorname{det}(T)^{-k-2 s} 2^{n(n+1-k-2 s)-l+1} i^{n k+l} \psi\left(\iota^{-1}(f)\right)(Z)
\end{aligned}
$$

and we have only to prove

$$
\begin{aligned}
\psi=\pi & \pi^{\frac{n(n+1)}{2}} \frac{(-1)^{\nu} \nu !}{(k+s+l-\nu-1)_{\nu+1}} \\
& \times \prod_{j=1}^{n-1} \frac{\Gamma(2 k+2 s-2 n+2 j-1)(2 k+2 s-n-2+j)_{l}}{(k+s-n-1+j) \Gamma(2 k+2 s+l+j-n-1)} .
\end{aligned}
$$

Here, we can write

$$
\begin{aligned}
& \psi(n, l, \nu, k+s-n-1) \\
& =\int_{S^{n}} \operatorname{det}\left(1_{n}-S \bar{S}\right)^{k+s-n-1-\nu}\left(\left(1_{n}-S \bar{S}\right)\left[{ }^{t} p_{n}\right]\right)^{l}\left(\bar{S}\left[{ }^{t} p_{n}\right]\right)^{\nu} \\
& \quad \times\left(\left(\frac{\partial}{\partial \bar{S}} \operatorname{det}\left(1_{n}-S \bar{S}\right)\right)\left[{ }^{t} p_{n}\right]\right)^{\nu} d S
\end{aligned}
$$

where $p_{n}^{(1, n)}=(1,0, \cdots, 0)$.

Let $\mu=k+s-n-1$. 
We put $S^{(n)}=\left(\begin{array}{cc}S_{1}^{(n-1)} & { }^{t} v^{(n-1,1)} \\ v^{(1, n-1)} & z\end{array}\right) . \quad$ By $1_{n-1}-S_{1} \bar{S}_{1}>0$, there exists $g \in G L(n-1, \mathbb{C})$ such that $1_{n-1}-S_{1} \bar{S}_{1}=g^{t} \bar{g}$. If we put $v=u^{t} g$, we get $d v=$ $|\operatorname{det} g|^{2} d u=\operatorname{det}\left(1_{n-1}-S_{1} \bar{S}_{1}\right) d u$. Moreover, we put

$$
\begin{gathered}
a=-\frac{1}{1-\bar{u}^{t} u}(<0), \quad b=-\frac{u^{t} g \bar{S}_{1} \bar{g}^{-1}{ }^{t} u}{1-\bar{u}^{t} u} \\
c=1-u^{t} g \bar{g}^{t} \bar{u}-u^{t} g \bar{S}_{1}{ }^{t} \bar{g}^{-1} g^{-1} S_{1} \bar{g}^{t} \bar{u}-\frac{\left|u^{t} g \bar{S}_{1}{ }^{t} \bar{g}^{-1} t u\right|^{2}}{\left|1-\bar{u}^{t} u\right|} .
\end{gathered}
$$

We note $|b|^{2}-a c=1$. Thus the condition $1_{n}-S \bar{S}>0$ is equivalent to the conditions

$$
1_{n-1}-S_{1} \bar{S}_{1}>0, \quad 1-\bar{u}^{t} u>0, \quad c+b \bar{z}+\bar{b} z+a z \bar{z}>0,
$$

and we get

$$
\begin{gathered}
\operatorname{det}\left(1_{n}-S \bar{S}\right)=\operatorname{det}\left(1_{n-1}-S_{1} \bar{S}_{1}\right)\left(1-\bar{u}^{t} u\right)(c+b \bar{z}+\bar{b} z+a z \bar{z}) \\
d S=\operatorname{det}\left(1_{n-1}-S_{1} \bar{S}_{1}\right) d S_{1} d u d z
\end{gathered}
$$

Therefore, we get

$$
\begin{aligned}
& \psi(n, l, \nu, \mu) \\
& =\int\left\{\operatorname{det}\left(1_{n-1}-S_{1} \bar{S}_{1}\right)^{\mu-\nu+1}\left(1-\bar{u}^{t} u\right)^{\mu}\right. \\
& \times\left(\left(g\left(1_{n-1}-{ }^{t} u \bar{u}\right)^{t} \bar{g}\right)\left[{ }^{t} p_{n-1}\right]\right)^{l}\left(\bar{S}_{1}\left[{ }^{t} p_{n-1}\right]\right)^{\nu} \\
& \times\left(\left(\frac{\partial}{\partial \bar{S}_{1}} \operatorname{det}\left(1_{n-1}-S_{1} \bar{S}_{1}\right)\right)\left[{ }^{t} p_{n-1}\right]\right)^{\nu} \\
& \left.\times\left\{\iint(c+b \bar{z}+\bar{b} z+a z \bar{z})^{\mu} d z\right\}\right\} d S_{1} d u
\end{aligned}
$$

By Hua $[14, \S 2.3]$, we have

$$
\iint(c+b \bar{z}+\bar{b} z+a z \bar{z})^{\mu} d z=\left(1-\bar{u}^{t} u\right)^{\mu+2} \frac{\pi}{\mu+1} .
$$

Using the equation above, we get

$$
\begin{aligned}
& \psi(n, l, \nu, \mu) \\
& =\frac{\pi}{\mu+1} \int_{S^{n-1}}\left\{\operatorname{det}\left(1_{n-1}-S_{1} \bar{S}_{1}\right)^{\mu-\nu+1}\left(\bar{S}_{1}\left[{ }^{t} p_{n-1}\right]\right)^{\nu}\right. \\
& \quad \times\left(\left(\frac{\partial}{\partial \bar{S}_{1}} \operatorname{det}\left(1_{n-1}-S_{1} \bar{S}_{1}\right)\right)\left[{ }^{t} p_{n-1}\right]\right)^{\nu} \\
& \left.\quad \times\left\{\int_{1-\bar{u}^{t} u>0}\left(1-\bar{u}^{t} u\right)^{2 \mu+2}\left(\left(g\left(1_{n-1}-{ }^{t} u \bar{u}\right)^{t} \bar{g}\right)\left[{ }^{t} p_{n-1}\right]\right)^{l} d u\right\}\right\} d S_{1} .
\end{aligned}
$$


By [9, Proposition 3.1], we have

$$
\begin{gathered}
\int\left(1-\bar{u}^{t} u\right)^{2 \mu+2}\left(\left(g\left(1_{n-1}-{ }^{t} u \bar{u}\right)^{t} \bar{g}\right)\left[{ }^{t} p_{n-1}\right]\right)^{l} d u \\
=d(n-1, l, 2 \mu+2)\left(\left(1_{n-1}-S_{1} \bar{S}_{1}\right)\left[{ }^{t} p_{n-1}\right]\right)^{l}
\end{gathered}
$$

where

$$
d(n, l, \mu)=\pi^{n} \frac{\Gamma(\mu+1)}{\Gamma(\mu+l+n+1)}(n+\mu)_{l} .
$$

Thus, we get

$$
\begin{aligned}
\psi(n, l, \nu, \mu) & =\frac{\pi}{\mu+1} \psi(n-1, l, \nu, \mu+1) d(n-1, l, 2 \mu+2) \\
& =\psi(1, l, \nu, \mu+n-1) \prod_{j=1}^{n-1} \frac{\pi}{(\mu+j)} d(n-j, l, 2 \mu+2 j) \\
& =\psi(1, l, \nu, \mu+n-1) \pi^{\frac{n(n+1)}{2}}-1 \prod_{j=1}^{n-1} \frac{\Gamma(2 \mu+2 j+1)(n+2 \mu+j)_{l}}{(\mu+j) \Gamma(2 \mu+j+l+n+1)},
\end{aligned}
$$

where

$$
\begin{aligned}
\psi(1, l, \nu, \mu+n-1) & =\iint_{S^{1}}(1-s \bar{s})^{\mu+n-1-\nu}(1-s \bar{s})^{l}(\bar{s})^{\nu}\left(\frac{\partial}{\partial \bar{s}}(1-s \bar{s})\right)^{\nu} d s \\
& =(-1)^{\nu} \frac{\nu !}{(\mu+n+l-\nu)_{\nu+1}} \pi .
\end{aligned}
$$

Now, by Proposition 2 and Theorem 1, we have

$$
\begin{aligned}
& \left(f,\left(L^{k, l} E_{k}^{2 n}\right)\left(\left(\begin{array}{cc}
-\bar{Z} & 0 \\
0 & *
\end{array}\right), \bar{s}\right)\right) \\
& =\sum_{\nu=0}^{\frac{l}{2}} \frac{a(l, \nu, k, s)}{(2 \pi i)^{l}} \sum_{r=1}^{n} \sum_{T \in \mathbb{T}^{(r)}}\left(f,\left(-\frac{1}{4}\right)^{\nu} \mathcal{P}_{\nu}\left(-\bar{Z}, *,\left(\begin{array}{cc}
0 & 0 \\
0 & T
\end{array}\right), \bar{s}\right)\right) \\
& =\sum_{\nu=0}^{\frac{l}{2}} \frac{a(l, \nu, k, s)}{(2 \pi i)^{l}} \sum_{T \in \mathbb{T}^{(n)}}\left(f,\left(-\frac{1}{4}\right)^{\nu} \mathcal{P}_{\nu}(-\bar{Z}, *, T, \bar{s})\right) .
\end{aligned}
$$

By (1.4), it equals

$$
\begin{aligned}
& 2^{n(n+1-k-2 s)-2 l+1} i^{n k} \pi^{\frac{n(n+1)}{2}}-l \prod_{j=1}^{n-1} \frac{\Gamma(2 k+2 s-2 n-1+2 j)(2 k+2 s-n-2+j)_{l}}{(k+s-n-1+j) \Gamma(2 k+2 s+l-n-1+j)} \\
& \quad \times\left\{\sum_{\nu=0}^{\frac{l}{2}} a(l, \nu, k, s) \frac{(-1)^{\nu} \nu !}{(k+s+l-\nu-1)_{\nu+1}}\right\} L(k+2 s, f)\left(\iota^{-1}(f)\right)(Z) .
\end{aligned}
$$


On the other hand, by (2.4), we get

$$
\sum_{\nu=0}^{\frac{l}{2}} a(l, \nu, k, s) \frac{(-1)^{\nu} \nu !}{(k+s+l-\nu-1)_{\nu+1}}=\sum_{\nu=0}^{\frac{l}{2}} b(l, \nu, k, s) \frac{(-1)^{\nu}}{k+s+l-\nu-1}
$$

Thus, by (1.5) and (2.5), we get

$$
\begin{aligned}
(f, & \left.\left(L^{k, l} E_{k}^{2 n}\right)\left(\left(\begin{array}{cc}
-\bar{Z} & 0 \\
0 & *
\end{array}\right), \bar{s}\right)\right) \\
= & 2^{n(n+1-k-2 s)-2 l+1} i^{n k} \pi^{\frac{n(n+1)}{2}}-l \\
& \quad \times \prod_{j=1}^{n-1} \frac{\Gamma(k+s-n) \Gamma(2 k+2 s+l-n-1)}{\Gamma(k+s-1) \Gamma(2 k+2 s+l-2)} \\
& \times\left\{\sum_{\nu=0}^{\frac{l}{2}} \frac{(-1)^{\nu}(2 k-2+2 v+2 j-2 n-1)}{\nu !(l-2 \nu) !(k-1+\nu)_{l-2 \nu}}(-s)_{\nu}\left(k+s+\frac{l}{2}-1\right)_{\frac{l}{2}-\nu}\right\} \\
& \times \zeta(2 s+k)^{-1} \prod_{j=1}^{n} \zeta(4 s+2 k-2 j)^{-1} D_{f}(2 s+k-n)\left(\iota^{-1}(f)\right)(Z) .
\end{aligned}
$$

Here, we prove the following:

Lemma 4. Let $k, l \in \mathbb{Z}, k, l>0$. For an indeterminate $X$,

$$
\begin{aligned}
& \sum_{\nu=0}^{\left[\frac{l}{2}\right]} \frac{(-1)^{\nu}(2 k-2+2 \nu)_{l-2 \nu}}{\nu !(l-2 \nu) !(k-1+\nu)_{l-\nu}}\left(-X+k-\frac{1}{2}\right)_{\nu}\left(X+\left[\frac{l+1}{2}\right]-\frac{1}{2}\right)_{\left[\frac{l}{2}\right]-\nu} \\
& =\frac{2^{l}}{l !} X_{\left[\frac{l}{2}\right]}
\end{aligned}
$$

Proof. We follow Zagier's method in [9, lemma 4.1].

We denote the left-hand side of $(3.3)$ by $P_{k, l}(X)$.

When we consider $P_{k, l}(X)$ a polynomial in $X$, the coefficient of the highest power of $X$ is

$$
\sum_{\nu=0}^{\left[\frac{l}{2}\right]} \frac{(2 k-2+2 \nu)_{l-2 \nu}}{\nu !(l-2 \nu) !(k-1+\nu)_{l-\nu}}
$$


Then we get

$$
\begin{aligned}
& \left(\begin{array}{c}
k+l-2 \\
l
\end{array}\right) \sum_{\nu=0}^{\left[\frac{l}{2}\right]} \frac{(2 k-2+2 \nu)_{l-2 \nu}}{\nu !(l-2 \nu) !(k-1+\nu)_{l-\nu}} \\
& =\frac{1}{l !} \sum_{\nu=0}^{\left[\frac{l}{2}\right]}\left(\begin{array}{c}
2 k+l-3 \\
l-2 \nu
\end{array}\right)\left(\begin{array}{c}
k+\nu-2 \\
k-2
\end{array}\right) \\
& =\frac{1}{l !} \operatorname{Res}_{x=0}\left[\frac{1}{\left(1-x^{2}\right)^{k-1}}(1+x)^{2 k+l-3} \frac{1}{x^{l+1}} d x\right]
\end{aligned}
$$

by putting $\quad t=\frac{x}{1+x}$

$$
\begin{aligned}
& =\frac{1}{l !} \operatorname{Res}_{t=0}\left[(1-2 t)^{-(k-1)} \frac{1}{t^{l+1}} d t\right] \\
& =\frac{2^{l}}{l !}\left(\begin{array}{c}
k+l-2 \\
l
\end{array}\right) .
\end{aligned}
$$

Thus, we have only to prove $P_{k, l}(-j)=0$ for $j \in \mathbb{Z}, 0 \leq j \leq\left[\frac{l}{2}\right]-1$.

We use induction on $l$ and $j$. We first prove $P_{k, l}(0)=0$ :

$$
P_{k, l}(0)=\frac{(2 k-2)_{l}}{(k-1)_{l}} \sum_{\nu=0}^{\left[\frac{l}{2}\right]} \frac{(-1)^{\nu}}{\nu !(l-2 \nu) ! 2^{2 \nu}}\left(\left[\frac{l+1}{2}\right]-\frac{1}{2}\right)_{\left[\frac{l}{2}\right]-\nu}
$$

For even $l$, by

$$
(l-2 \nu) !=\frac{2^{l-2 \nu}\left(\frac{l}{2}-\nu\right) !\left(\frac{1}{2}\right)_{\frac{l}{2}}}{\left(\frac{1-l}{2}\right)_{\nu}(-1)^{\nu}}
$$

we have

$$
\begin{aligned}
P_{k, l}(0) & =\frac{(2 k-2)_{l}}{(k-1)_{l} 2^{l}\left(\frac{1}{2}\right)_{\frac{l}{2}}} \sum_{\nu=0}^{\frac{l}{2}} \frac{1}{\nu !\left(\frac{l}{2}-\nu\right) !}\left(\frac{1-l}{2}\right)_{\nu}\left(\frac{l-1}{2}\right)_{\frac{l}{2}-\nu} \\
& =0
\end{aligned}
$$

For odd $l$, by

$$
(l-2 \nu) !=\frac{2^{l-2 \nu}\left(\frac{l-1}{2}-\nu\right) !\left(\frac{1}{2}\right)_{\frac{l+1}{2}}}{\left(-\frac{l}{2}\right)_{\nu}(-1)^{\nu}}
$$


we have

$$
\begin{aligned}
P_{k, l}(0) & =\frac{(2 k-2)_{l}}{(k-1)_{l} 2^{l}\left(\frac{1}{2}\right)} \sum_{\frac{l+1}{2}}^{\frac{l-1}{2}} \frac{1}{\nu !\left(\frac{l-1}{2}-\nu\right) !}\left(-\frac{l}{2}\right)_{\nu}\left(\frac{l}{2}\right)_{\frac{l-1}{2}-\nu} \\
& =0
\end{aligned}
$$

Next, we suppose $P_{k, l^{\prime}}\left(-j^{\prime}\right)=0$ for any $k, j^{\prime}, l^{\prime}$ such that $j^{\prime}<j, l^{\prime}<l$.

For even $l$, if we note

$$
\left(k-\frac{1}{2}+j\right)_{\nu}=\nu\left(k-\frac{1}{2}+j\right)_{\nu-1}+\left(k-\frac{1}{2}+j-1\right)_{\nu}
$$

and

$$
\left(\frac{l-1}{2}-j\right)_{\frac{l}{2}-\nu}=-\frac{l-2 \nu}{2}\left(\frac{l-1}{2}-(j-1)\right)_{\frac{l}{2}-\nu-1}+\left(\frac{l-1}{2}-(j-1)\right)_{\frac{l}{2}-\nu}
$$

we have

$$
\begin{aligned}
P_{k, l}(-j) & \sum_{\nu=0}^{\frac{l}{2}} \frac{(-1)^{\nu}(2 k-2+2 \nu)_{l-2 \nu}}{\nu !(l-2 \nu) !(k-1+\nu)_{l-\nu}}\left(k-\frac{1}{2}+j-1\right)_{\nu}\left(\frac{l-1}{2}-(j-1)\right)_{\frac{l}{2}-\nu} \\
& +\sum_{\nu=1}^{\frac{l}{2}} \frac{(-1)^{\nu}(2 k-2+2 \nu)_{l-2 \nu}}{(\nu-1) !(l-2 \nu) !(k-1+\nu)_{l-\nu}}\left(k-\frac{1}{2}+j\right)_{\nu-1}\left(\frac{l-1}{2}-(j-1)\right)_{\frac{l}{2}-\nu} \\
& -\sum_{\nu=0}^{\frac{l}{2}-1} \frac{(-1)^{\nu}(2 k-2+2 \nu)_{l-2 \nu}}{2(\nu) !(l-1-2 \nu) !(k-1+\nu)_{l-\nu}}\left(k-\frac{1}{2}+j\right)_{\nu}\left(\frac{l-1}{2}-(j-1)\right)_{\frac{l}{2}-\nu-1} \\
= & P_{k, l}(-(j-1)) \\
& -\sum_{\nu=0}^{\frac{l}{2}-1}\left\{\frac{(-1)^{\nu}(2 k+2 \nu)_{l-2 \nu-2}}{2(\nu) !(l-1-2 \nu) !(k-1+\nu)_{l-\nu}}\{2(k+\nu-1)(l-1-2 \nu)\right. \\
= & P_{k, l}(-(j-1))-P_{k+1, l-1}(-(j-1)) \\
= & 0 .
\end{aligned}
$$

In the same way, for odd $l$, we have

$$
P_{k, l}(-j)=P_{k, l}(-(j-1))-\frac{2}{l-2 j} P_{k+1, l-1}(-(j-1))+\frac{1}{l-2 j} P_{k, l}(-j) .
$$


Thus, Lemma 4 is proved.

By Lemma 4, we get

$$
\begin{aligned}
& \left(f,\left(L^{k, l} E_{k}^{2 n}\right)\left(\left(\begin{array}{cc}
-\bar{Z} & 0 \\
0 & *
\end{array}\right), \bar{s}\right)\right) \\
& =\frac{1}{(k)_{l} l !} 2^{n(n+1-k-2 s)-l+1} i^{n k} \pi^{\frac{n(n+1)}{2}}-l \prod_{j=1}^{n-1} \frac{\Gamma(2 k+2 s+2 j-2 n-1)}{\Gamma(2 k+2 s+j-n-2)} \\
& \quad \times \frac{\Gamma\left(k+s+\frac{l}{2}-1\right) \Gamma\left(k+s+\frac{l}{2}-\frac{1}{2}\right) \Gamma(k+s-n) \Gamma(2 k+2 s+l-n-1)}{\Gamma(k+s) \Gamma\left(k+s-\frac{1}{2}\right) \Gamma(k+s-1) \Gamma(2 k+2 s+l-2)} \\
& \quad \times \zeta(2 s+k)^{-1} \prod_{j=1}^{n} \zeta(4 s+2 k-2 j)^{-1} D_{f}(2 s+k-n)\left(\iota^{-1}(f)\right)(Z) .
\end{aligned}
$$

Combining this with (1.6), we obtain

$$
\begin{aligned}
& \left(f,\left(L^{k, l} \mathbb{E}_{k}^{2 n}\right)\left(\left(\begin{array}{cc}
-\bar{Z} & 0 \\
0 & *
\end{array}\right), \frac{\bar{s}+n}{2}\right)\right) \\
& =\frac{1}{(k)_{l} l !} 2^{1-l} i^{n k} \pi^{-\frac{1}{2} n^{2}+k n+\frac{1}{2} \varepsilon} \gamma(s) \\
& \quad \times \Gamma_{\mathbb{R}}(s+\varepsilon) \Gamma_{\mathbb{C}}(s+k+l-1) \prod_{j=2}^{n} \Gamma_{\mathbb{C}}(s+k-j) D_{f}(s)\left(\iota^{-1}(f)\right)(Z),
\end{aligned}
$$

where $\varepsilon$ and $\gamma(s)$ are of the form (1.8) and (1.9), respectively. Here, we put

$$
\Lambda(s, f):=\Gamma_{\mathbb{R}}(s+\varepsilon) \Gamma_{\mathbb{C}}(s+k+l-1) \prod_{j=2}^{n} \Gamma_{\mathbb{C}}(s+k-j) D_{f}(s) .
$$

On the other hand, it follows from (1.4.1) and (1.4.2) that $\left(f,\left(L^{k, l} \mathbb{E}_{k}^{2 n}\right)\left(\left(\begin{array}{cc}-\bar{Z} & 0 \\ 0 & *\end{array}\right), \bar{s}\right)\right)$ is invariant under $s \mapsto \frac{n+1}{2}-s$ and that it is an entire function in $s$. Thus, we have:

TheOrem 2. Let $k, l \in 2 \mathbb{Z}, k>0, l \geq 0$. If $f \in S_{k, l}^{n}\left(\operatorname{sym}^{l}\left(V_{2}\right)\right)$ is an eigenform,

(i)

$$
\begin{aligned}
(f, & \left.\left(L^{k, l} \mathbb{E}_{k}^{2 n}\right)\left(\left(\begin{array}{cc}
-\bar{Z} & 0 \\
0 & *
\end{array}\right), \frac{\bar{s}+n}{2}\right)\right) \\
& =\frac{1}{(k)_{l} l !} 2^{1-l} i^{n k} \pi^{-\frac{1}{2} n^{2}+k n+\frac{1}{2} \varepsilon} \gamma(s) \Lambda(s, f)\left(\iota^{-1}(f)\right)(Z)
\end{aligned}
$$


or equivalently,

$$
\begin{aligned}
\alpha_{k, l}^{n}(s) \zeta(s & +n) \prod_{j=0}^{n-1} \zeta(2 s+2 j)\left(f,\left(L^{k, l} E_{k}^{2 n}\right)\left(\left(\begin{array}{cc}
-\bar{Z} & 0 \\
0 & *
\end{array}\right), \frac{\bar{s}+n-k}{2}\right)\right) \\
& =\Lambda(s, f)\left(\iota^{-1}(f)\right)(Z),
\end{aligned}
$$

where

$$
\begin{aligned}
\alpha_{k, l}^{n}(s):=(k)_{l} l ! & 2^{\frac{n^{2}-3 n}{2}+s n-1+l} i^{n k} \pi^{-\left(n+\frac{1}{2}\right) s-n k-\frac{n+\varepsilon}{2}} \\
& \times \Gamma_{n}\left(\frac{s+n+k}{2}\right) \Gamma_{n}\left(\frac{s+k}{2}\right) \Gamma\left(\frac{s+\varepsilon}{2}\right) .
\end{aligned}
$$

(ii) $\Lambda(s, f)$ has a meromorphic continuation to the whole s-plane and satisfies the functional equation

$$
\Lambda(s, f)=\Lambda(1-s, f)
$$

REMARK. For $\left(\lambda_{1}, \lambda_{2}, \cdots, \lambda_{n}\right) \in \mathbb{Z}^{n}$ and $\lambda_{1} \geq \lambda_{2} \geq \cdots \geq \lambda_{n} \geq 0$, let $\rho \sim\left(\lambda_{1}, \lambda_{2}, \cdots, \lambda_{n}\right)$ be an irreducible rational representation of $G L(n, \mathbb{C})$ with a representation space $\mathcal{V}$. Suppose that $f \in S_{\rho}^{n}(\mathcal{V})$ is an eigenform. Then, it is expected that completed Dirichlet series

$$
\Lambda(s, f):=\Gamma_{\mathbb{R}}(s+\varepsilon) \prod_{j=1}^{n} \Gamma_{\mathbb{C}}\left(s+\lambda_{j}-j\right) D_{f}(s)
$$

should satisfy a functional equation.

\subsection{Poles of standard $L$-functions}

Theorem 3. Let $k, l \in 2 \mathbb{Z}, k>0, l \geq 0$ and $k>n$. If $f \in S_{k, l}^{n}\left(\operatorname{sym}^{l}\left(V_{2}\right)\right)$ is an eigenform, $\Lambda(s, f)$ is holomorphic except for possible simple poles at $s=0$ and $s=1$; it has a pole at $s=0$ (or equivalently, $s=1$ ) if and only if $f \in$ $B_{k, l}^{n}(2 n) \cap S_{k, l}^{n}\left(\operatorname{sym}^{l}\left(V_{2}\right)\right)$.

COROLlaRY. Under the assumption of Theorem 3 , suppose $n \not \equiv 0 \bmod 4$. Then $\Lambda(s, f)$ is entire.

Proof of Theorem 3. Theorem 3 is proved in the same way as that by Mizumoto [21, Theorem 1]:

Let $\Gamma_{*}$ be a finite-index subgroup of $\Gamma^{n}$. A function $g$ from $\mathfrak{H}_{n}$ to $\mathbb{C}$ is called a $C^{\infty}$-modular form of weight $k$ (i.e., $\rho=\operatorname{det}^{k} \otimes \operatorname{sym}^{0}$ ) with respect to $\Gamma_{*}$ if it is a $C^{\infty}$-function and satisfies

$$
(g \mid M)(Z):=\operatorname{det}(C Z+D)^{-k} g(M\langle Z\rangle)=g(Z)
$$


for all $M=\left(\begin{array}{cc}* & * \\ C^{(n)} & D^{(n)}\end{array}\right) \in \Gamma_{*}$. Note that this notation $\mid$ is compatible with the one in $\S 1.1$.

Let $\Gamma_{j}(j=1,2)$ be finite-index subgroups of $\Gamma^{n}$ such that $\Gamma_{1} \subset \Gamma_{2}$. Suppose $g$ is a $C^{\infty}$-modular form of weight $k$ with respect to $\Gamma_{1}$. Then

$$
\operatorname{Tr}\left(\Gamma_{1}, \Gamma_{2} ; g\right)(Z):=\sum_{\gamma \in \Gamma_{1} \backslash \Gamma_{2}}(g \mid \gamma)(Z)
$$

is a $C^{\infty}$-modular form of weight $k$ with respect to $\Gamma_{2}$.

For $n, N \in \mathbb{Z}, k \in 2 \mathbb{Z}, n, N, k>0$ and $Z \in \mathfrak{H}_{n}$, we put

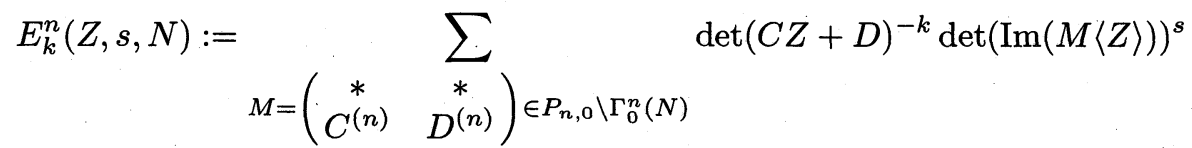

for $k+2 \operatorname{Re}(s)>n+1$, which has a meromorphic continuation to the whole $s$-plane by [20]. In particular,

$$
E_{k}^{n}(Z, s, 1)=E_{k}^{n}(Z, s)
$$

By Shimura [25, Proposition 2.1],

$$
\operatorname{Tr}\left(\Gamma_{0}^{n}(N), \Gamma^{n} ; E_{k}^{n}(Z, s, N)\right)=E_{k}^{n}(Z, s),
$$

so

$$
\begin{aligned}
& E_{k}^{2 n}\left(\mathfrak{Z}, \frac{s+n-k}{2}\right) \\
& \quad=\operatorname{Tr}\left(J^{-1} \Gamma_{0}^{2 n}(N) J, \Gamma^{2 n} ; E_{k}^{2 n}\left(*, \frac{s+n-k}{2}, N\right) \mid J\right)
\end{aligned}
$$

where $\mathfrak{Z} \in \mathfrak{H}_{2 n}$ and $J:=\left(\begin{array}{cc}0 & 1_{2 n} \\ -1_{2 n} & 0\end{array}\right)$.

Suppose $k>n$ and $N$ : even. Using the same notation as in [11], [21], let

$$
\begin{aligned}
& D^{2 n}\left(\mathfrak{Z}, \frac{s+n-k}{2} ; k, 1, N\right) \\
& \quad:=\beta_{k}^{n}(s) \zeta_{N}(s+n) \prod_{j=0}^{n-1} \zeta_{N}(2 s+2 j)\left(E_{k}^{2 n}\left(*, \frac{s+n-k}{2}, N\right) \mid J\right)(\mathfrak{Z}),
\end{aligned}
$$

where

$$
\zeta_{N}(s):=\prod_{\substack{p \mid N \\ p: \text { prime }}}\left(1-p^{-s}\right) \zeta(s)
$$


and

$$
\begin{aligned}
\beta_{k}^{n}(s):= & 2^{s-1} \pi^{-\frac{1}{2}} \Gamma\left(\frac{s+\varepsilon}{2}\right) \Gamma\left(\frac{s+n+k}{2}\right) \Gamma\left(\frac{s+k-n+1}{2}\right) \\
& \times \prod_{j=1}^{n-1} \Gamma(s+k+n-2 j) .
\end{aligned}
$$

Now, $\alpha_{k, l}^{n}(s)$ in Theorem 2 (i) and

$$
\beta_{k}^{n}(s) \prod_{p \mid N}\left\{\left(1-p^{-s-n}\right) \prod_{j=0}^{n-1}\left(1-p^{-2 s-2 j}\right)\right\}
$$

are holomorphic and non-zero in the region $\operatorname{Re}(s)>0$. So

$$
g(s):=\left(\beta_{k}^{n}(s) \prod_{p \mid N}\left\{\left(1-p^{-s-n}\right) \prod_{j=0}^{n-1}\left(1-p^{-2 s-2 j}\right)\right\}\right)^{-1}
$$

is also holomorphic and non-zero in the region $\operatorname{Re}(s)>0$.

Hence, by (3.7) and (3.8), we get

$$
\begin{aligned}
& \zeta(s+n) \prod_{j=0}^{n-1} \zeta(2 s+2 j) E_{k}^{2 n}\left(\mathfrak{Z} \frac{s+n-k}{2}\right) \\
& \quad=g(s) \operatorname{Tr}_{J}^{N}\left(D^{2 n}\left(*, \frac{s+n-k}{2} ; k, 1, N\right)\right)(\mathfrak{Z})
\end{aligned}
$$

where

$$
\operatorname{Tr}_{J}^{N}(*):=\operatorname{Tr}\left(J^{-1} \Gamma_{0}^{2 n}(N) J, \Gamma^{2 n} ; *\right) .
$$

Thus (3.6) may also be written as follows:

$$
\begin{aligned}
& \Lambda(s, f)\left(\iota^{-1}(f)\right)(Z) \\
& \quad=\alpha_{k, l}^{n}(s) g(s)\left(f,\left(L^{k, l} \operatorname{Tr}_{J}^{N}\left(D^{2 n}\left(*, \frac{\bar{s}+n-k}{2} ; k, 1, N\right)\right)\right)\left(\begin{array}{cc}
-\bar{Z} & 0 \\
0 & *
\end{array}\right)\right) .
\end{aligned}
$$

A result of Feit [11, Theorem 9.1] tells us that

$$
D^{2 n}\left(*, \frac{s+n-k}{2} ; k, 1, N\right)
$$


for even $N$ is holomorphic in $s$ except for a possible simple pole at $s=1$; moreover, it is entire if $n$ is odd.

On the other hand, it follows from (3.9) and (1.4.2) that convergence of

$$
\left(f,\left(L^{k, l} \operatorname{Tr}_{J}^{N}\left(D^{2 n}\left(*, \frac{\bar{s}+n-k}{2} ; k, 1, N\right)\right)\right)\left(\begin{array}{cc}
-\bar{Z} & 0 \\
0 & *
\end{array}\right)\right)
$$

in $s$ is the same as that of (3.11) in $s$. Hence the integral representation (3.10) shows that $\Lambda(s, f)$ is holomorphic for $\operatorname{Re}(s)>0$ except for a possible simple pole at $s=1$ and that the pole does not appear if $n$ is odd. By (1.3.1), $\Lambda(s, f)$ has a pole at $s=1$ exactly when $n \equiv 0 \bmod 4$ and $f \in B_{k, l}^{n}(2 n) \cap S_{k, l}^{n}\left(\operatorname{sym}^{l}\left(V_{2}\right)\right)$. Combining these facts with the functional equation in Theorem 2 (ii), we obtain Theorem 3.

\section{References}

[1] A. N. Andrianov, Euler products corresponding to Siegel modular forms of genus 2, Russian Math. Surveys, 29 (1974), 45-116; English translation.

[2] A. N. Andrianov, The multiplicative arithmetic of Siegel modular forms, Russian Math. Surveys, 34 (1979), 75-148; English translation.

[ 3 ] A. N. Andrianov and V.L. Kalinin, On the analytic properties of standard zeta function of Siegel modular forms, Math. USSR-Sb., 35 (1979), 1-17; English translation.

[ 4 ] T. Arakawa, Vector valued Siegel's modular forms of degree two and the associated Andrianov $L$-functions, Manuscripta Math., 44 (1983), 155-185.

[ 5 ] S. Böcherer, Über die Fourier-Jacobi-Entwicklung Siegelscher Eisensteinreihen, Math. Z., 183 (1983), 21-46.

[6] S. Böcherer, Über die Fourier-Jacobi-Entwicklung Siegelscher Eisensteinreihen II, Math. Z., 189 (1985), 81-110.

[ 7 ] S. Böcherer, Über die Funktionalgleichung automorpher $L$-functionen zur Siegelschen Modulgruppe, J. Reine Angew. Math., 362 (1985), 146-168.

[ 8 ] S. Böcherer, Ein Rationalitätssatz für formale Heckereihen zur Siegelschen Modulgruppe, Abh. Math. Sem. Univ. Hamburg, 56 (1986), 35-47.

[ 9 ] S. Böcherer, T. Satoh and T. Yamazaki, On the pullback of a differential operator and its application to vector valued Eisenstein series, Comment. Math. Univ. St. Pauli., 42 (1992), 1-22.

[10] S. A. Evdokimov, A characterization of the Maass space of Siegel cusp forms of second degree, Math. USSR-Sb., 40 (1981), 125-133; English translation.

[11] P. Feit, Poles and residues of Eisenstein series for symplectic and unitary groups, Mem. Amer. Math. Soc., 61 no. 346, Providence, Rhode Island, 1986.

[12] P. B. Garrett, Pullbacks of Eisenstein series ; applications, Automorphic Forms of Several Variables, Progress in Math., 46, 114-137, Birkhäuser, Boston-Basel-Stuttgart (1984).

[13] R. Godement, Fonctions Automorphes, Séminaire H. Cartan, E.N.S, 1957/1958.

[14] L. K. Hua, Harmonic analysis of functions of several complex variables in the classical domains, Trans. Amer. Math. Soc., 6, Providence, Rhode Island, 1963.

[15] V. L. Kalinin, Eisenstein series on the symplectic group, Math. USSR-Sb., 32 (1977), 449476; English translation.

[16] H. Klingen, Über Poincarésche Reihen zur Siegelschen Modulgruppe, Math. Ann., 168 (1967), 157-170. 
[17] H. Klingen, Zum Darstellungssatz für Siegelsche Modulformen, Math. Z., 102 (1967), 3043.

[18] R. P. Langlands, Problems in the theory of automorphic forms, Lecture Notes in Math., 170, 18-86, Springer, Berlin-Heidelberg-New York 1970.

[19] R. P. Langlands, Euler products, Yale Univ. Press, 1971.

[20] R. P. Langlands, On the functional equations satisfied by Eisenstein series, Lecture Notes in Math., 544, Springer, Berlin-Heidelberg-New York, 1976.

[21] S. Mizumoto, Poles and residues of standard $L$-functions attached to Siegel modular forms, Math. Ann., 289 (1991), 589-612.

[22] S. Mizumoto, Eisenstein series for Siegel modular groups, preprint.

[23] T. Oda, On the poles of Andrianov $L$-functions, Math. Ann., 256 (1981), 323-340.

[24] I. Piatetski-Shapiro and S. Rallis, $L$-functions for the classical groups, Lecture Notes in Math., 1254, Springer, Berlin-Heidelberg-New York, 1987.

[25] G. Shimura, On Eisenstein series, Duke Math. J., 50 (1983), 417-476.

[26] T. Sugano, On holomorphic cusp forms on quaternion unitary groups of degree 2, J. Fac. Sci. Univ. Tokyo, 31 (1984), 521-568.

[27] R. Weissauer, Vectorwertige Siegelsche Modulformen Kleinen Gewichtes, J. Reine Angew. Math., 343 (1983), 184-202.

[28] R. Weissauer, Stabile Modulformen und Eisensteinreihen, Lecture Notes in Math., 1219, Springer, Berlin-Heidelberg-New York, 1986.

\author{
Department OF MATHEMATiCs \\ KEIO UNIVERSITY \\ 3-14-1 HIYOSHI KOHOKU-KU \\ YOKOHAMA, 223, JAPAN
}

